\title{
The multicomponent 2D Toda hierarchy: dispersionless limit
}

\author{
Manuel Mañas and Luis Martínez Alonso \\ Departamento de Física Teórica II, Universidad Complutense \\ 28040-Madrid, Spain \\ emails: manuel.manas@fis.ucm.es, luism@fis.ucm.es
}

November 21, 2018

\begin{abstract}
The factorization problem of the multi-component 2D Toda hierarchy is used to analyze the dispersionless limit of this hierarchy. A dispersive version of the Whitham hierarchy defined in terms of scalar Lax and Orlov-Schulman operators is introduced and the corresponding additional symmetries and string equations are discussed. Then, it is shown how KP and Toda pictures of the dispersionless Whitham hierarchy emerge in the dispersionless limit. Moreover, the additional symmetries and string equations for the dispersive Whitham hierarchy are studied in this limit.
\end{abstract}

\section{Introduction}

In [1] the theory of the multi-component Toda hierarchy [2] was analyzed from the point of view of a factorization problem

$$
g=W^{-1} \bar{W}
$$

in an infinite-dimensional group and a natural formulation of the additional symmetries and the string equations of the hierarchy was given. In the present work we use this formulation to study the dispersionless limit of the solutions of (1). As it is known in the theory of random matrix models [3]-[5], the study the large $N$ limit can be performed in terms of the dispersionless limit of the string equations satisfied by the solution of the underlying integrable system. Notice that in recent years the formalism of string equations [6] for dispersionless integrable systems [7] has been much developed [8]. Our present work is motivated by the applications of multi-component integrable hierarchies [2, 9] to the study of the large $N$ limit of the two-matrix model [10]-[13, as well as models of random matrices with external source and non-intersecting Brownian motions [14-[19. A common feature of these models is that they have an associated family of multiple orthogonal polynomials which is in turn characterized by a matrix Riemann-Hilbert $(\mathrm{MRH})$ problem which is a basic ingredient to analyze the large $N$ limit 19-22]. On the other hand, MRH problems also provide solutions of reductions of multi-component integrable hierarchies of KP or Toda type. These reductions correspond to solutions of factorization problems (11) constrained by certain types of string equations.

In our analysis we introduce matrix wave functions and scalar Lax and Orlov-Schulman operators [23] associated to the solutions of (11). We prove that the rows of the matrix wave functions satisfy auxiliary linear systems involving the scalar Lax operators, which constitute the dispersive versions of the genus zero dispersionless Whitham hierarchies 24. In order to study the dispersionless limit, we assume the TakasakiTakebe quasi-classical ansatz 25, 26] for the rows of the matrix wave functions. Thus, we prove that in the dispersionless limit the auxiliary linear systems reduce to systems of Hamilton-Jacobi equations that are shown to be equivalent to the dispersionless Whitham hierarchies. In particular, two natural pictures (KP and Toda types) of the dispersionless Whitham hierarchies emerge in our analysis. An important advantage of our approach is that it yields a natural method for characterizing string equations and additional symmetries in the dispersionless limit. In particular, we characterize the dispersive analogues of the soluble string equations discussed in [27.

The layout of the paper is as follows. In $\S 1.1$ we present a summary of the relevant parts of [1] needed in the subsequent analysis. Then, in $\S 2$ we discuss the dispersive Whitham hierarchies. We introduce a set of scalar 
Lax and Orlov-Schulman operators, and vector wave functions to deduce the corresponding auxiliary linear systems, as well as additional symmetries and string equations of dispersive type. Finally, in $\S 3$ we discuss the aforementioned dispersionless limits. We find the Hamilton-Jacobi type equations, and then derive the KP and Toda pictures of the dispersionless Whitham hierarchy. We conclude the paper by considering the dispersionless counterparts of dispersive string equations.

\subsection{Reminder}

As in our previous work [1] we only consider formal series expansions in the Lie group theoretic set up without any assumption on their convergency. Let us remind some notations and results from [1. Given Lie algebras $\mathfrak{g}_{1} \subset \mathfrak{g}_{2}$, and $X, Y \in \mathfrak{g}_{2}$ then $X=Y+\mathfrak{g}_{1}$ means $X-Y \in \mathfrak{g}_{1}$. For any Lie groups $G_{1} \subset G_{2}$ and $a, b \in G_{2}$ then $a=G_{2} \cdot b$ stands for $a \cdot b^{-1} \in G_{2}$.

Let $M_{N}(\mathbb{C})$ denote the associative algebra of complex $N \times N$ complex matrices we will consider the linear space of sequences $f: \mathbb{Z} \rightarrow M_{N}(\mathbb{C})$. The shift operator $\Lambda$ acts on these sequences as $(\Lambda f)(n):=f(n+1)$. A sequence $X: \mathbb{Z} \rightarrow M_{N}(\mathbb{C})$ acts by left multiplication in this space of sequences, and therefore we may consider operators of the type $X \Lambda^{j},\left(X \Lambda^{j}\right)(f)(n):=X(n) \cdot f(n+j)$.

Moreover, defining the product $\left(X(n) \Lambda^{i}\right) \cdot\left(Y(n) \Lambda^{j}\right):=X(n) Y(n+i) \Lambda^{i+j}$ and extending it linearly we have that the set $\mathfrak{g}$ of Laurent series in $\Lambda$ is an associative algebra, which under the standard commutator is a Lie algebra.

This Lie algebra has the following important splitting

$$
\mathfrak{g}=\mathfrak{g}_{+} \dot{+} \mathfrak{g}_{-}
$$

where

$$
\mathfrak{g}_{+}=\left\{\sum_{j \geq 0} X_{j}(n) \Lambda^{j}, \quad X_{j}(n) \in M_{N}(\mathbb{C})\right\}, \quad \mathfrak{g}_{-}=\left\{\sum_{j<0} X_{j}(n) \Lambda^{j}, \quad X_{j}(n) \in M_{N}(\mathbb{C})\right\},
$$

are Lie subalgebras of $\mathfrak{g}$ with trivial intersection.

The group of linear invertible elements in $\mathfrak{g}$ will be denoted by $G$ and has $\mathfrak{g}$ as its Lie algebra, then the splitting (2) leads us to consider the following factorization of $g \in G$

$$
g=g_{-}^{-1} \cdot g_{+}, \quad g_{ \pm} \in G_{ \pm}
$$

where $G_{ \pm}$have $\mathfrak{g}_{ \pm}$as their Lie algebras. Explicitly, $G_{+}$is the set of invertible linear operators of the form $\sum_{j \geq 0} g_{j}(n) \Lambda^{j}$; while $G_{-}$is the set of invertible linear operators of the form $1+\sum_{j<0} g_{j}(n) \Lambda^{j}$.

Now we introduce two sets of indexes, $\mathbb{S}=\{1, \ldots, N\}$ and $\overline{\mathbb{S}}=\{\overline{1}, \ldots, \bar{N}\}$, of the same cardinality $N$. In what follows we will use letters $k, l$ and $\bar{k}, \bar{l}$ to denote elements in $\mathbb{S}$ and $\overline{\mathbb{S}}$, respectively. Furthermore, we will use letters $a, b, c$ to denote elements in $\mathcal{S}:=\mathbb{S} \cup \overline{\mathbb{S}}$.

We define the following operators $W_{0}, \bar{W}_{0} \in G$

$$
\begin{aligned}
W_{0} & :=\sum_{k=1}^{N} E_{k k} \Lambda^{s_{k}} \mathrm{e}^{\sum_{j=0}^{\infty} t_{j k} \Lambda^{j}}, \\
\bar{W}_{0} & :=\sum_{k=1}^{N} E_{k k} \Lambda^{-s_{\bar{k}}} \mathrm{e}^{\sum_{j=1}^{\infty} t_{j \bar{k}} \Lambda^{-j}}
\end{aligned}
$$

where $s_{a} \in \mathbb{Z}, t_{j a} \in \mathbb{C}$ are deformation parameters, that in the sequel will play the role of discrete and continuous times, respectively. Given an element $g \in G$ and a set of deformation parameters $\boldsymbol{s}=\left(s_{a}\right)_{a \in \mathcal{S}}, \boldsymbol{t}=\left(t_{j a}\right)_{a \in \mathcal{S}, j \in \mathbb{N}}$ we will consider the factorization problem

$$
S(\boldsymbol{s}, \boldsymbol{t}) \cdot W_{0} \cdot g=\bar{S}(\boldsymbol{s}, \boldsymbol{t}) \cdot \bar{W}_{0}, \quad S \in G_{-} \text {and } \bar{S} \in G_{+},
$$

and will confine ourselves to the zero charge sector $|s|:=\sum_{a \in \mathcal{S}} s_{a}=0$. We define the dressing or Sato operators $W, \bar{W}$ as follows

$$
W:=S \cdot W_{0}, \quad \bar{W}:=\bar{S} \cdot \bar{W}_{0},
$$


so that the factorization problem in $G$ reads

$$
W \cdot g=\bar{W}
$$

Observe that $S, \bar{S}$ have expansions of the form

$$
\begin{aligned}
& S=\mathbb{I}_{n}+\varphi_{1}(n) \Lambda^{-1}+\varphi_{2}(n) \Lambda^{-2}+\cdots \in G_{-}, \\
& \bar{S}=\bar{\varphi}_{0}(n)+\bar{\varphi}_{1}(n) \Lambda+\bar{\varphi}_{2}(n) \Lambda^{2}+\cdots \in G_{+} .
\end{aligned}
$$

The Lax operators $L, \bar{L}, C_{k k}, \bar{C}_{k k} \in \mathfrak{g}$ are defined by

$$
\begin{aligned}
L & :=W \cdot \Lambda \cdot W^{-1}, & \bar{L} & :=\bar{W} \cdot \Lambda \cdot \bar{W}^{-1}, \\
C_{k k} & :=W \cdot E_{k k} \cdot W^{-1}, & \bar{C}_{k k} & :=\bar{W} \cdot E_{k k} \cdot \bar{W}^{-1}
\end{aligned}
$$

and have the following expansions

$$
\begin{aligned}
L & =\Lambda+u_{1}(n)+u_{2}(n) \Lambda^{-1}+\cdots, & \bar{L}^{-1} & =\bar{u}_{0}(n) \Lambda^{-1}+\bar{u}_{1}(n)+\bar{u}_{2}(n) \Lambda+\cdots, \\
C_{k k} & =E_{k k}+C_{k k, 1}(n) \Lambda^{-1}+C_{k k, 2}(n) \Lambda^{-2}+\cdots, & \overline{\mathcal{C}}_{k k} & =\bar{C}_{k k, 0}(n)+\bar{C}_{k k, 1}(n) \Lambda+\bar{C}_{k k, 2}(n) \Lambda^{2}+\cdots .
\end{aligned}
$$

Now we introduce some further notation

1.

$$
\partial_{j a}:=\frac{\partial}{\partial t_{j a}}, \quad \text { for } a=\mathcal{S} \text { and } j=1,2, \ldots
$$

2. Given $K=(a, b)$ the basic charge preserving shift operators $T_{K}$ are defined as follows

$$
\left(T_{K} f\right)\left(s_{a}, s_{b}\right):=f\left(s_{a}+1, s_{b}-1\right) .
$$

We define the Orlov-Schulman operators [23] for the multi-component 2D Toda hierarchy by

$$
M:=W n W^{-1}, \quad \bar{M}:=\bar{W} n \bar{W}^{-1} .
$$

One proves at once that

- The Orlov-Schulman operators satisfy the following commutation relations

$$
[L, M]=L, \quad\left[L, C_{k k}\right]=0, \quad[\bar{L}, \bar{M}]=\bar{L}, \quad\left[\bar{L}, \bar{C}_{k k}\right]=0
$$

- The following expansions hold

$$
\begin{array}{ll}
M=\mathcal{M}+\sum_{k=1}^{N} C_{k k}\left(s_{k}+\sum_{j=1}^{\infty} j t_{j k} L^{j}\right), \quad \mathcal{M}=n+\mathfrak{g}_{-} \\
\bar{M}=\overline{\mathcal{M}}-\sum_{k=1}^{N} \bar{C}_{k k}\left(s_{\bar{k}}+\sum_{j=1}^{\infty} j t_{j \bar{k}} \bar{L}^{-j}\right), & \overline{\mathcal{M}}=n+\mathfrak{g}_{+} \Lambda .
\end{array}
$$

\subsubsection{Additional symmetries}

Suppose that the operator $g$ in (8) depends on an additional parameter $6 \in \mathbb{C}$. Then, the basic objects of the multi-component Toda hierarchy inherit a dependence on 6 . For convenience and for the time being we use the following equivalent factorization problem

$$
W \cdot h=\bar{W} \cdot \bar{h},
$$

with

$$
g=h \cdot \bar{h}^{-1}
$$


Observe that

$$
\begin{aligned}
\partial_{6} W \cdot W^{-1}+W\left(\partial_{6} h \cdot h^{-1}\right) W^{-1} & =\partial_{6} S \cdot S^{-1}+W\left(\partial_{6} h \cdot h^{-1}\right) W^{-1} \\
& =\partial_{6} \bar{S} \cdot \bar{S}^{-1}+\bar{W}\left(\partial_{6} \bar{h} \cdot \bar{h}^{-1}\right) \bar{W}^{-1}=\partial_{6} \bar{W} \cdot \bar{W}^{-1}+\bar{W}\left(\partial_{6} \bar{h} \cdot \bar{h}^{-1}\right) \bar{W}^{-1} .
\end{aligned}
$$

Now, let us suppose that $h$ and $\bar{h}$ satisfy

$$
\partial_{6} h \cdot h^{-1}=F^{(0)}=\sum_{l=1}^{N} F_{l}(n, \Lambda) E_{l l}, \quad \partial_{6} \bar{h} \cdot \bar{h}^{-1}=\bar{F}^{(0)}=\sum_{l=1}^{N} \bar{F}_{l}(n, \Lambda) E_{l l},
$$

then from (17)we get

$$
\partial_{6} W \cdot W^{-1}=\partial_{6} S \cdot S^{-1}=-H_{-}, \quad \partial_{6} \bar{W} \cdot \bar{W}^{-1}=\partial_{6} \bar{S} \cdot \bar{S}^{-1}=H_{+}, \quad H_{ \pm} \in \mathfrak{g}_{ \pm} .
$$

where

$$
H:=F-\bar{F}, \quad F:=\sum_{l=1}^{N} F_{l}(M, L) C_{l l}, \quad \bar{F}:=\sum_{l=1}^{N} \bar{F}_{l}(\bar{M}, \bar{L}) \bar{C}_{l l} .
$$

Hence it follows that

Proposition 1. Given a dependence on an additional parameter 6 according to (16), (18) and (19) then

1. The dressing operators $W$ and $\bar{W}$ satisfy

$$
\partial_{6} W=-H_{-} \cdot W, \quad \partial_{6} \bar{W}=H_{+} \cdot \bar{W},
$$

2. The Lax and Orlov-Schulman operators satisfy

$$
\begin{aligned}
& \partial_{6} L=\left[-H_{-}, L\right], \quad \partial_{6} M=\left[-H_{-}, M\right], \quad \partial_{6} C_{k k}=-\left[H_{-}, C_{k k}\right], \\
& \partial_{6} \bar{L}=\left[H_{+}, \bar{L}\right], \quad \partial_{6} \bar{M}=\left[H_{+}, \bar{M}\right], \quad \partial_{6} \bar{C}_{k k}=\left[H_{+}, \bar{C}_{k k}\right] .
\end{aligned}
$$

A key observation is

Proposition 2. Given operators $R, \bar{R} \in \mathfrak{g}$ satisfying $R \cdot g=\bar{R}$ and such that

$$
\begin{aligned}
& R W_{0}^{-1} \in \mathfrak{g}_{-}, \\
& \bar{R} \bar{W}_{0}^{-1} \in \mathfrak{g}_{+} .
\end{aligned}
$$

Then $R=\bar{R}=0$

\subsection{Wave functions}

The wave functions of the multi-component 2D Toda hierarchy are defined by

$$
\psi=W \cdot \chi, \quad \bar{\psi}=\bar{W} \cdot \chi .
$$

where

$$
\chi(z):=\left\{z^{n} \mathbb{I}_{N}\right\}_{n \in \mathbb{Z}}
$$

Note that $\Lambda \chi=z \chi$. The following asymptotic expansions are a consequence of (9)

$$
\begin{aligned}
& \psi=z^{n}\left(\mathbb{I}_{N}+\varphi_{1}(n) z^{-1}+\cdots\right) \psi_{0}(z), \quad \psi_{0}:=\sum_{k=1}^{N} E_{k k} z^{s_{k}} \mathrm{e}^{\sum_{j=1}^{\infty} t_{j k} z^{j}}, \quad z \rightarrow \infty, \\
& \bar{\psi}=z^{n}\left(\bar{\varphi}_{0}(n)+\bar{\varphi}_{1}(n) z+\cdots\right) \bar{\psi}_{0}(z), \quad \bar{\psi}_{0}:=\sum_{k=1}^{N} E_{k k} z^{-s_{\bar{k}}} \mathrm{e}^{\sum_{j=1}^{\infty} t_{j \bar{k}} z^{-j}}, \quad z \rightarrow 0 .
\end{aligned}
$$


Proposition 3. 1. Given operators of the form

$$
F:=\sum_{k=1}^{N} F_{k} C_{k k}, \quad F_{k}:=\sum_{i \geq 0, j \in \mathbb{Z}} F_{k i j} M^{i} L^{j}, \quad \bar{F}:=\sum_{k=1}^{N} F_{\bar{k}} \bar{C}_{k k}, \quad F_{\bar{k}}:=\sum_{i \geq 0, j \in \mathbb{Z}} F_{\bar{k} i j} \bar{M}^{i} \bar{L}^{j},
$$

with complex-valued scalar coefficients, we have

$$
F(\psi)=(\psi) \sum_{k=1}^{N} \overleftarrow{F_{k}\left(z \frac{\mathrm{d}}{\mathrm{d} z}, z\right)} E_{k k}, \quad \bar{F}(\bar{\psi})=(\bar{\psi}) \sum_{k=1}^{N} \overleftarrow{F_{\bar{k}}\left(z \frac{\mathrm{d}}{\mathrm{d} z}, z\right)} E_{k k} .
$$

where

$$
\left(\psi \overleftarrow{F_{k}\left(z \frac{\mathrm{d}}{\mathrm{d} z}, z\right)}:=\sum_{i \geq 0, j \in \mathbb{Z}} F_{k i j} z^{j}\left(z \frac{\mathrm{d}}{\mathrm{d} z}\right)^{i}(\psi), \quad(\bar{\psi}) \overleftarrow{F_{\bar{k}}\left(z \frac{\mathrm{d}}{\mathrm{d} z}, z\right)}:=\sum_{i \geq 0, j \in \mathbb{Z}} \bar{F}_{k i j} z^{j}\left(z \frac{\mathrm{d}}{\mathrm{d} z}\right)^{i}(\bar{\psi})\right.
$$

2. Given operators

$$
\begin{array}{llll}
P:=\sum_{k=1}^{N} P_{k} C_{k k}, & P_{k}:=\sum_{i \geq 0, j \in \mathbb{Z}} P_{k i j} M^{i} L^{j}, & Q:=\sum_{k=1}^{N} Q_{k} C_{k k}, & Q_{k}:=\sum_{i \geq 0, j \in \mathbb{Z}} Q_{k i j} M^{i} L^{j}, \\
\bar{P}:=\sum_{k=1}^{N} P_{\bar{k}} \bar{C}_{k k}, & P_{\bar{k}}:=\sum_{i \geq 0, j \in \mathbb{Z}} P_{\bar{k} i j} \bar{M}^{i} \bar{L}^{j}, & \bar{Q}:=\sum_{k=1}^{N} Q_{\bar{k}} \bar{C}_{k k}, & Q_{\bar{k}}:=\sum_{i \geq 0, j \in \mathbb{Z}} \bar{Q}_{\bar{k} i j} \bar{M}^{i} \bar{L}^{j},
\end{array}
$$

with complex-valued scalar coefficients, we have

$$
P Q(\psi)=\sum_{k=1}^{N}\left((\psi) \overleftarrow{P_{k}\left(z \frac{\mathrm{d}}{\mathrm{d} z}, z\right)}\right) \overleftarrow{Q_{k}\left(z \frac{\mathrm{d}}{\mathrm{d} z}, z\right)}, \quad \bar{P} \bar{Q}(\bar{\psi})=\sum_{k=1}^{N}\left((\bar{\psi}) \overleftarrow{P_{\bar{k}}\left(z \frac{\mathrm{d}}{\mathrm{d} z}, z\right)}\right) \overleftarrow{Q_{\bar{k}}\left(z \frac{\mathrm{d}}{\mathrm{d} z}, z\right)} .
$$

Proof. 1. It is easy to find from (10) and (15) that

$$
\begin{aligned}
& M^{i} L^{j} C_{k k}(\psi)=W n^{i} \Lambda^{j} E_{k k}(\chi)=W\left(\left\{n^{i} z^{n+j} \mathbb{I}_{N}\right\}_{n \in \mathbb{Z}}\right) E_{k k}, \\
& \bar{M}^{i} \bar{L}^{j} \bar{C}_{k k}(\bar{\psi})=\bar{W} n^{i} \Lambda^{j} E_{k k}(\chi)=\bar{W}\left(\left\{n^{i} z^{n+j} \mathbb{I}_{N}\right\}_{n \in \mathbb{Z}}\right) E_{k k} .
\end{aligned}
$$

Now observe that the action of $X=\sum_{j^{\prime} \in \mathbb{Z}} X_{j^{\prime}} \Lambda^{j^{\prime}}$ on $\left\{n^{i} z^{n+j}\right\}_{n \in \mathbb{Z}}$ is

$$
X\left(\left\{n^{i} z^{n+j}\right\}_{n \in \mathbb{Z}}\right)=\left\{\sum_{j^{\prime} \in \mathbb{Z}} X_{j^{\prime}}(n)\left(n+j^{\prime}\right)^{i} z^{n+j+j^{\prime}}\right\}_{n \in \mathbb{Z}}
$$

or equivalently

$$
z^{j}\left(z \frac{\mathrm{d}}{\mathrm{d} z}\right)^{i}(X \cdot \chi)=\left\{z^{j}\left(z \frac{\mathrm{d}}{\mathrm{d} z}\right)^{i}\left(\sum_{j^{\prime} \in \mathbb{Z}} X_{j^{\prime}}(n) z^{n+j^{\prime}}\right)\right\}_{n \in \mathbb{Z}} .
$$

Thus, the formulae

$$
M^{i} L^{j} C_{k k}(\psi)=z^{j}\left(z \frac{\mathrm{d}}{\mathrm{d} z}\right)^{i}(\psi) E_{k k}, \quad \quad \bar{M}^{i} \bar{L}^{j} \bar{C}_{k k}(\bar{\psi})=z^{j}\left(z \frac{\mathrm{d}}{\mathrm{d} z}\right)^{i}(\bar{\psi}) E_{k k},
$$

hold.

2. It is a consequence of the identities

$$
\begin{aligned}
M^{i_{1}} L^{j_{1}} M^{i_{2}} L^{j_{2}} & =M^{i_{1}}\left(M+j_{1}\right)^{i_{2}} L^{j_{1}+j_{2}}, \\
\bar{M}^{i_{1}} \bar{L}^{j_{1}} \bar{M}^{i_{2}} \bar{L}^{j_{2}} & =\bar{M}^{i_{1}}\left(\bar{M}+j_{1}\right)^{i_{2}} \bar{L}^{j_{1}+j_{2}}, \\
z^{j_{2}}\left(z \frac{\mathrm{d}}{\mathrm{d} z}\right)^{i_{2}} z^{j_{1}}\left(z \frac{\mathrm{d}}{\mathrm{d} z}\right)^{i_{1}} & =z^{j_{1}+j_{2}}\left(z \frac{\mathrm{d}}{\mathrm{d} z}+j_{1}\right)^{i_{2}}\left(z \frac{\mathrm{d}}{\mathrm{d} z}\right)^{i_{1}},
\end{aligned}
$$

for any $i_{1}, i_{2} \geq 0$ and $j_{1}, j_{2} \in \mathbb{Z}$. Therefore,

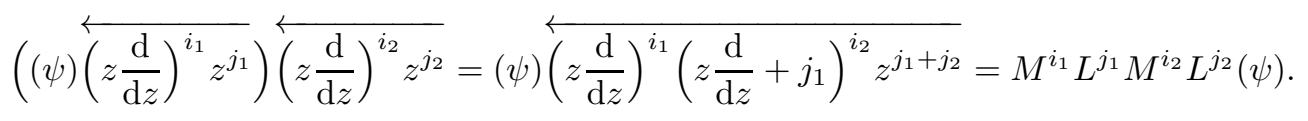




\section{The dispersive Whitham hierarchies}

As we will see certain families of equations of the multi-component 2D Toda hierarchy, associated with any given row of the dressing operators, become the Whitham hierarchies under appropriate dispersionless limits. Consequently, these families will be referred to as the dispersive Whitham hierarchies.

For simplicity and without loss of generality, we will work with the first row of the dressing operators. It will be useful to introduce the following shift operators

$$
\mathcal{T}_{a}:=\left\{\begin{array}{ll}
T_{\left(1, a_{0}\right)}, & a=1, \\
T_{(a, 1)}, & a \neq 1
\end{array} \quad \overline{\mathcal{T}}_{a}:= \begin{cases}T_{\left(\overline{1}, a_{0}\right)}, & a=\overline{1}, \\
T_{(a, \overline{1})}, & a \neq \overline{1},\end{cases}\right.
$$

where for the cases $a=1$ and $a=\overline{1}$, the index $a_{0}$ stands for any fixed elements in $\mathcal{S}-\{1\}$ and $\overline{\mathcal{S}}-\{\overline{1}\}$, respectively. These two types of shift operators, that we refer as bared and unbared, lead to two algebras of shift operators, and also to two different families of Hamilton-Jacobi equations, see (47) and (48). We also define the scalar dressing operators

$$
\begin{aligned}
\mathscr{K}_{a} & := \begin{cases}1+\varphi_{1,11} \mathcal{T}_{1}^{-1}+\varphi_{2,11} \mathcal{T}_{l}^{-2}+\cdots, & a=1 \\
\varphi_{1,1 k}+\varphi_{2,1 k} \mathcal{T}_{k}^{-1}+\cdots, & a=k \neq 1, \\
\bar{\varphi}_{0,1 k}+\bar{\varphi}_{1,1 k} \mathcal{T}_{\bar{k}}^{-1}+\cdots, & a=\bar{k},\end{cases} \\
\overline{\mathscr{K}}_{a} & := \begin{cases}1+\varphi_{1,11} \overline{\mathcal{T}}_{1}^{-1}+\varphi_{2, l l} \overline{\mathcal{T}}_{1}^{-2}+\cdots, & a=1, \\
\varphi_{1, l k}+\varphi_{2, l k} \overline{\mathcal{T}}_{k}^{-1}+\cdots, & a=k \neq 1 \\
\bar{\varphi}_{0,1 k}+\bar{\varphi}_{1,1 k} \overline{\mathcal{T}}_{\bar{k}}^{-1}+\cdots, & a=\bar{k},\end{cases}
\end{aligned}
$$

where $\varphi_{i}, \bar{\varphi}_{i}$ are the matrix coefficients of (9).

Thus, we may now introduce the associated scalar Lax operators

$$
\begin{aligned}
& \mathscr{L}_{a}:=\mathscr{K}_{a} \circ \mathcal{T}_{a} \circ \mathscr{K}_{a}^{-1}=\mathscr{W}_{a} \circ \mathcal{T}_{a} \circ \mathscr{W}_{a}^{-1}= \begin{cases}\mathcal{T}_{1}+L_{1,0}+L_{1,-1} \mathcal{T}_{1}^{-1}+\cdots, & a=1, \\
L_{a, 1} \mathcal{T}_{a}+L_{a, 0}+L_{a,-1} \mathcal{T}_{a}^{-1}+\cdots, & a \neq 1\end{cases} \\
& \overline{\mathscr{L}}_{a}:=\overline{\mathscr{K}}_{a} \circ \overline{\mathcal{T}}_{a} \circ \overline{\mathscr{K}}_{a}^{-1}=\overline{\mathscr{W}}_{a} \circ \overline{\mathcal{T}}_{a} \circ \overline{\mathscr{W}}_{a}^{-1}= \begin{cases}\overline{\mathcal{T}}_{1}+\bar{L}_{1,0}+\bar{L}_{1,-1} \overline{\mathcal{T}}_{1}^{-1}+\cdots, & a=1, \\
\bar{L}_{a, 1} \overline{\mathcal{T}}_{a}+\bar{L}_{a, 0}+\bar{L}_{a,-1} \overline{\mathcal{T}}_{a}^{-1}+\cdots, & a \neq 1,\end{cases}
\end{aligned}
$$

where

$$
\begin{aligned}
\mathscr{W}_{a}:=\mathscr{K}_{a} \circ \mathscr{W}_{0, a}, & \mathscr{W}_{0, a}:=\exp \left(\mathscr{T}_{a}\right), & \mathscr{T}_{a}:=\sum_{j=1}^{\infty} t_{j a} \mathcal{T}_{a}^{j}, \\
\overline{\mathscr{W}}_{a}:=\overline{\mathscr{K}}_{a} \circ \overline{\mathscr{W}}_{0, a}, & \overline{\mathscr{W}}_{0, a}:=\exp \left(\overline{\mathscr{T}}_{a}\right), & \overline{\mathscr{T}}_{a}:=\sum_{j=1}^{\infty} t_{j a} \overline{\mathcal{T}}_{a}^{j} .
\end{aligned}
$$

Similarly, we define the corresponding scalar Orlov-Schulman operators by

$$
\mathscr{M}_{a}:=n-\nu_{a}+\operatorname{sg}(a) \mathscr{W}_{a} \circ s_{a} \circ \mathscr{W}_{a}^{-1}, \quad \quad \overline{\mathscr{M}}_{a}:=n-\nu_{a}+\operatorname{sg}(a) \overline{\mathscr{W}}_{a} \circ s_{a} \circ \overline{\mathscr{W}}_{a}^{-1},
$$

where

$$
\operatorname{sg}(a):=\left\{\begin{array}{ll}
1, & a \in \mathbb{S}, \\
-1, & a \in \bar{S},
\end{array} \quad \nu_{a}:= \begin{cases}1, & a \in \mathbb{S}-\{1\} \\
0, & a \notin \mathbb{S}-\{1\} .\end{cases}\right.
$$

From the identities

$$
\left[\mathcal{T}_{a}, \operatorname{sg}(a) s_{a}\right]=\operatorname{sg}(a) \mathcal{T}_{a}, \quad\left[\overline{\mathcal{T}}_{a}, \operatorname{sg}(a) s_{a}\right]=\operatorname{sg}(a) \overline{\mathcal{T}}_{a},
$$

it follows that

$$
\left[\mathscr{L}_{a}, \mathscr{M}_{a}\right]=\operatorname{sg}(a) \mathscr{L}_{a}, \quad\left[\overline{\mathscr{L}}_{a}, \overline{\mathscr{M}}_{a}\right]=\operatorname{sg}(a) \overline{\mathscr{L}}_{a}
$$


Proposition 4. The Orlov-Schulman operators satisfy

$$
\begin{aligned}
& \mathscr{M}_{a}=n-\nu_{a}+\operatorname{sg}(a)\left(s_{a}+\sum_{j=1}^{\infty} j t_{j a} \mathscr{L}_{a}^{j}+\sum_{i=1}^{\infty} m_{a i} \mathcal{T}_{a}^{-i}\right), \\
& \overline{\mathscr{M}}_{a}:=n-\nu_{a}+\operatorname{sg}(a)\left(s_{a}+\sum_{j=1}^{\infty} j t_{j a} \overline{\mathscr{L}}_{a}^{j}+\sum_{i=1}^{\infty} \bar{m}_{a i} \overline{\mathcal{T}}_{a}^{-i}\right) .
\end{aligned}
$$

Proof. These formulae follow from

$$
\mathscr{W}_{0, a} s_{a} \mathscr{W}_{0, a}^{-1}=s_{a}+\left[\mathscr{T}_{a}, s_{a}\right]=s_{a}+\sum_{j=1}^{\infty} j \mathcal{T}_{a}^{j}, \quad \overline{\mathscr{W}}_{0, a} s_{a} \overline{\mathscr{W}}_{0, a}^{-1}=s_{a}+\left[\overline{\mathscr{T}}_{a}, s_{a}\right]=s_{a}+\sum_{j=1}^{\infty} j \overline{\mathcal{T}}_{a}^{j},
$$

and the fact that there are expansions of the form

$$
\mathscr{K}_{a} s_{a} \mathscr{K}_{a}^{-1}=s_{a}+\sum_{i=1}^{\infty} m_{a i} \mathcal{T}_{a}^{-i}, \quad \quad \overline{\mathscr{K}}_{a} s_{a} \overline{\mathscr{K}}_{a}^{-1}=s_{a}+\sum_{i=1}^{\infty} \bar{m}_{a i} \overline{\mathcal{T}}_{a}^{-i} .
$$

We further introduce the vector wave functions

$$
\Psi_{a}:= \begin{cases}\psi_{1 k}, & a=k \\ \bar{\psi}_{1 k}, & a=\bar{k}\end{cases}
$$

Proposition 5. We have the identities

$$
\left[F_{a}\left(\mathscr{M}_{a}, \mathscr{L}_{a}\right)\right]\left(\Psi_{a}\right)=\left[F_{a}\left(\overline{\mathscr{M}}_{a}, \overline{\mathscr{L}}_{a}\right)\right]\left(\Psi_{a}\right)=\left(\Psi_{a}\right) \overleftarrow{F_{a}\left(z \frac{\mathrm{d}}{\mathrm{d} z}, z^{\mathrm{sg} a}\right)}= \begin{cases}E_{11} F_{k}(M, L) C_{k k}(\psi), & a=k \\ E_{11} F_{\bar{k}}\left(\bar{M}, \bar{L}^{-1}\right) \bar{C}_{k k}(\bar{\psi}), & a=\bar{k}\end{cases}
$$

Proof. From the definitions (44),(5) and (7)

$$
\begin{aligned}
& W_{1 k} n^{i} \Lambda^{j}=S_{1 k} W_{0, k k} n^{i} \Lambda^{j}=S_{1 k}\left(W_{0, k k} n W_{0, k k}^{-1}\right)^{i} \Lambda^{j} W_{0, k k}=S_{1 k}\left(n+s_{k}+\sum_{j^{\prime}=1}^{\infty} j^{\prime} t_{j^{\prime} k} \Lambda^{j^{\prime}}\right)^{i} \Lambda^{j} W_{0, k k}, \\
& \bar{W}_{1 k} n^{i} \Lambda^{j}=\bar{S}_{1 k} \bar{W}_{0, k k} n^{i} \Lambda^{j}=\bar{S}_{1 k}\left(\bar{W}_{0, k k} n \bar{W}_{0, k k}^{-1}\right)^{i} \Lambda^{j} \bar{W}_{0, k k}=\bar{S}_{1 k}\left(n-s_{\bar{k}}-\sum_{j^{\prime}=1}^{\infty} j^{\prime} t_{j^{\prime} \bar{k}} \Lambda^{-j^{\prime}}\right)^{i} \Lambda^{j} \bar{W}_{0, k k},
\end{aligned}
$$

Now, observe that

$$
\begin{aligned}
\Lambda^{-1}\left(n+s_{k}\right) W_{0, k k} & =\mathcal{T}_{k}^{-1}\left(\left(n+s_{k}\right) W_{0, k k}\right)=\overline{\mathcal{T}}_{k}^{-1}\left(\left(n+s_{k}\right) W_{0, k k}\right), \\
\Lambda\left(n-s_{\bar{k}}\right) \bar{W}_{0, k k} & =\mathcal{T}_{\bar{k}}^{-1}\left(\left(n-s_{\bar{k}}\right) \bar{W}_{0, k k}\right)=\overline{\mathcal{T}}_{\bar{k}}^{-1}\left(\left(n-s_{\bar{k}}\right) \bar{W}_{0, k k}\right),
\end{aligned}
$$

together with Proposition 3 imply the result.

\subsection{Auxiliary linear systems}

Our next analysis uses the following complex algebras

$$
\mathfrak{t}_{a}:=\left\{\sum_{j \in \mathbb{Z}} c_{j} \mathcal{T}_{a}^{j}\right\}, \quad \overline{\mathfrak{t}}_{a}:=\left\{\sum_{j \in \mathbb{Z}} c_{j} \overline{\mathcal{T}}_{a}^{j}\right\},
$$


and their subalgebras

$$
\begin{aligned}
\begin{cases}\mathfrak{t}_{a,+}=\mathfrak{t}_{a,>}:=\left\{\sum_{j>0} c_{j} \mathcal{T}_{a}^{j}\right\} \quad \mathfrak{t}_{a, \leq}:=\left\{\sum_{j \leq 0} c_{j} \mathcal{T}_{a}^{j}\right\}, \quad a \neq 1 \\
\mathfrak{t}_{1,+}=\mathfrak{t}_{1, \geq}:=\left\{\sum_{j \geq 0} c_{j} \mathcal{T}_{1}^{j}\right\} \quad \mathfrak{t}_{1,<}:=\left\{\sum_{j<0} c_{j} \mathcal{T}_{1}^{j}\right\}\end{cases} \\
\left\{\begin{array}{lll}
\overline{\mathfrak{t}}_{a,+}=\overline{\mathfrak{t}}_{a,>}:=\left\{\sum_{j>0} c_{j}\left(\overline{\mathcal{T}}_{a}^{j}-1\right)\right\}, & \overline{\mathfrak{t}}_{a,<}:=\left\{\sum_{j<0} c_{j}\left(\overline{\mathcal{T}}_{a}^{j}-1\right)\right\}, & a \neq 1, \overline{1} \\
\overline{\mathfrak{t}}_{1,+}=\overline{\mathfrak{t}}_{1, \geq}:=\left\{\sum_{j \geq 0} c_{j} \overline{\mathcal{T}}_{1}^{j}\right\}, & \overline{\mathfrak{t}}_{1,<}:=\left\{\sum_{j<0} c_{j} \overline{\mathcal{T}}_{1}^{j}\right\}, & \\
\overline{\mathfrak{t}}_{\overline{1},+}=\overline{\mathfrak{t}}_{\overline{1},>}:=\left\{\sum_{j>0} c_{j}\left(\overline{\mathcal{T}}_{\overline{1}}^{j}-1\right)\right\} & \overline{\mathfrak{t}}_{\overline{1},<}:=\left\{\sum_{j<0} c_{j}\left(\overline{\mathcal{T}}_{\overline{1}}^{j}-1\right)\right\}, & a^{\prime} \neq 1, \\
\overline{\mathfrak{t}}_{\overline{1},+}=\overline{\mathfrak{t}}_{\overline{1},>}:=\left\{\sum_{j>0} c_{j} \overline{\mathcal{T}}_{\overline{1}}^{j}\right\} & \overline{\mathfrak{t}}_{\overline{1}, \leq}:=\left\{\sum_{j \leq 0} c_{j} \overline{\mathcal{T}}_{\overline{1}}^{j}\right\}, & a^{\prime}=1 .
\end{array}\right.
\end{aligned}
$$

We will denote by $\left(T_{a,+}, T_{a,<}, T_{a,>}, T_{a, \leq}, T_{a, \geq}\right)$ the projections of an operator $T_{a}$ induced by the corresponding splittings.

The following important result links the operators $\left(\mathscr{M}_{k}, \mathscr{L}_{k}\right)$ with the operators $(M, L)$. Here the splittings for each shift algebra $\mathfrak{t}_{a}$ or $\overline{\mathfrak{t}}_{a}$ are those indicated by (38) and (39),

Proposition 6. The following relations hold

$$
\begin{aligned}
& \left\{\begin{array}{l}
F\left(\mathscr{M}_{k}, \mathscr{L}_{k}\right)_{+}\left(E_{11} W\right)=F\left(\overline{\mathscr{M}}_{k}, \overline{\mathscr{L}}_{k}\right)_{+}\left(E_{11} W\right)=E_{11}\left(F(M, L) C_{k k}\right)_{+} W, \\
F\left(\mathscr{M}_{k}, \mathscr{L}_{k}\right)_{+}\left(E_{11} \bar{W}\right)=F\left(\overline{\mathscr{M}}_{k}, \overline{\mathscr{L}}_{k}\right)_{+}\left(E_{11} \bar{W}\right)=E_{11}\left(F(M, L) C_{k k}\right)_{+} \bar{W},
\end{array}\right. \\
& \left\{\begin{array}{l}
F\left(\mathscr{M}_{\bar{k}}, \mathscr{L}_{\bar{k}}\right)_{+}\left(E_{11} W\right)=F\left(\overline{\mathscr{M}}_{\bar{k}}, \overline{\mathscr{L}}_{\bar{k}}\right)_{+}\left(E_{11} W\right)=E_{11}\left(F\left(\bar{M}, \bar{L}^{-1}\right) \bar{C}_{k k}\right)_{-} W, \\
F\left(\mathscr{M}_{\bar{k}}, \mathscr{L}_{\bar{k}}\right)_{+}\left(E_{11} \bar{W}\right)=F\left(\overline{\mathscr{M}}_{\bar{k}}, \overline{\mathscr{L}}_{\bar{k}}\right)_{+}\left(E_{11} \bar{W}\right)=E_{11}\left(F\left(\bar{M}, \bar{L}^{-1}\right) \bar{C}_{k k}\right)_{-} \bar{W} .
\end{array}\right.
\end{aligned}
$$

Proof. See Appendix B.

If we set $F(x, y)=y^{j}$ in Proposition 6 and recall that

$$
\partial_{j a} W=B_{j a} W, \quad \partial_{j a} \bar{W}=B_{j a} \bar{W},
$$

with $B_{j k}=\left(C_{k k} L^{j}\right)_{+}, B_{j \bar{k}}=\left(\bar{C}_{k k} \bar{L}^{-j}\right)_{-}$[1] we deduce

Theorem 1. The following scalar linear systems hold

$$
\partial_{j a}\left(E_{11} W\right)=\left(\mathscr{L}_{a}^{j}\right)_{+}\left(E_{11} W\right)=\left(\overline{\mathscr{L}}_{a}^{j}\right)_{+}\left(E_{11} W\right), \quad \partial_{j a}\left(E_{11} \bar{W}\right)=\left(\mathscr{L}_{a}^{j}\right)_{+}\left(E_{11} \bar{W}\right)=\left(\overline{\mathscr{L}}_{a}^{j}\right)_{+}\left(E_{11} \bar{W}\right)
$$

The linear system (42) determines a set of commuting flows for $(W, \bar{W})$ which, as we will show in the next Section, leads to the Whitham hierarchy in the dispersionless limit. For that reason this system will be referred to as the dispersive Whitham hierarchy of flows.

\subsection{Additional symmetries and string equations}

Using Proposition 1 we deduce the following results on the additional symmetries

Proposition 7. Given an additional symmetry

$$
\begin{aligned}
& \partial_{6} E_{11} W=-E_{11}\left(\sum_{k=1}^{N}\left(F_{k}(M, L) C_{k k}-F_{\bar{k}}\left(\bar{M}, \bar{L}^{-1}\right) \bar{C}_{k k}\right)\right)_{-} \cdot W \\
& \partial_{6} E_{11} \bar{W}=E_{11}\left(\sum_{k=1}^{N}\left(F_{k}(M, L) C_{k k}-F_{\bar{k}}\left(\bar{M}, \bar{L}^{-1}\right) \bar{C}_{k k}\right)\right)_{+} \cdot \bar{W}
\end{aligned}
$$


then we have

$$
\begin{aligned}
\partial_{b}\left(\Psi_{a}\right) & =-F_{a}\left(\mathscr{M}_{a}, \mathscr{L}_{a}\right)\left(\Psi_{a}\right)+\left(\sum_{a^{\prime} \in \mathcal{S}} F_{a^{\prime}}\left(\mathscr{M}_{a^{\prime}}, \mathscr{L}_{a^{\prime}}\right)_{+}\right)\left(\Psi_{a}\right) \\
& =-F_{a}\left(\overline{\mathscr{M}}_{a}, \overline{\mathscr{L}}_{a}\right)\left(\Psi_{a}\right)+\left(\sum_{a^{\prime} \in \mathcal{S}} F_{a^{\prime}}\left(\overline{\mathscr{M}}_{a^{\prime}}, \overline{\mathscr{L}}_{a^{\prime}}\right)_{+}\right)\left(\Psi_{a}\right) .
\end{aligned}
$$

Proof. From (43) we get

$$
\begin{aligned}
& \partial_{6} E_{11} W=-\sum_{k=1}^{N} E_{11} F_{k}(M, L) C_{k k} \cdot W+E_{11}\left[\left(\sum_{k=1}^{N} F_{k}(M, L) C_{k k}\right)_{+}+\left(\sum_{k=1}^{N} \bar{F}_{k}\left(\bar{M}, \bar{L}^{-1}\right) \bar{C}_{k k}\right)_{-}\right] \cdot W, \\
& \partial_{6} E_{11} \bar{W}=-\sum_{k=1}^{N} E_{11} \bar{F}_{\bar{k}}\left(\bar{M}, \bar{L}^{-1}\right) \bar{C}_{k k} \cdot \bar{W}+E_{11}\left[\left(\sum_{k=1}^{N} F_{k}(M, L) C_{k k}\right)_{+}+\left(\sum_{k=1}^{N} F_{\bar{k}}\left(\bar{M}, \bar{L}^{-1}\right) \bar{C}_{k k}\right)_{-}\right] \cdot \bar{W} .
\end{aligned}
$$

Now, from Propositions 5] and [6] we conclude that

$$
\begin{aligned}
\partial_{6}\left(E_{11} W\right) & =-\sum_{k=1}^{N} F_{k}\left(\mathscr{M}_{k}, \mathscr{L}_{k}\right)\left(W_{1 k}\right) E_{1 k}+\left(\sum_{k=1}^{N}\left(F_{k}\left(\mathscr{M}_{k}, \mathscr{L}_{k}\right)_{+}+F_{\bar{k}}\left(\mathscr{M}_{\bar{k}}, \mathscr{L}_{\bar{k}}\right)_{+}\right)\left(E_{11} W\right)\right. \\
& =-\sum_{k=1}^{N} F_{k}\left(\overline{\mathscr{M}}_{k}, \overline{\mathscr{L}}_{k}\right)\left(W_{1 k}\right) E_{1 k}+\left(\sum_{k=1}^{N}\left(F_{k}\left(\overline{\mathscr{M}}_{k}, \overline{\mathscr{L}}_{k}\right)_{+}+F_{\bar{k}}\left(\overline{\mathscr{M}}_{\bar{k}}, \overline{\mathscr{L}}_{\bar{k}}\right)_{+}\right)\left(E_{l l} W\right)\right. \\
\partial_{6}\left(E_{11} \bar{W}\right) & =-\sum_{k=1}^{N} \bar{F}_{k}\left(\mathscr{M}_{k}, \mathscr{L}_{k}\right)\left(\bar{W}_{1 k}\right) E_{l k}+\left(\sum_{k=1}^{N}\left(F_{k}\left(\mathscr{\mathscr { M }}_{k}, \mathscr{L}_{k}\right)_{+}+F_{\bar{k}}\left(\mathscr{M}_{\bar{k}}, \mathscr{L}_{\bar{k}}\right)_{+}\right)\left(E_{11} \bar{W}\right)\right. \\
& =-\sum_{k=1}^{N} \bar{F}_{k}\left(\overline{\mathscr{M}}_{k}, \overline{\mathscr{L}}_{k}\right)\left(\bar{W}_{1 k}\right) E_{1 k}+\left(\sum_{k=1}^{N}\left(F_{k}\left(\overline{\mathscr{M}}_{k}, \overline{\mathscr{L}}_{k}\right)_{+}+F_{\bar{k}}\left(\overline{\mathscr{M}}_{\bar{k}}, \overline{\mathscr{L}}_{\bar{k}}\right)_{+}\right)\left(E_{11} \bar{W}\right) .\right.
\end{aligned}
$$

and the result follows.

As a consequence we have

Proposition 8. If the string equation

$$
E_{11} \sum_{k=1}^{N} F_{k}(M, L) C_{k k}=E_{11} \sum_{k=1}^{N} F_{\bar{k}}\left(\bar{M}, \bar{L}^{-1}\right) \bar{C}_{k k}
$$

is satisfied, then

$$
F_{a}\left(\mathscr{M}_{a}, \mathscr{L}_{a}\right)\left(\Psi_{a}\right)=\left(\sum_{a^{\prime} \in \mathcal{S}} F_{a^{\prime}}\left(\mathscr{M}_{a^{\prime}}, \mathscr{L}_{a^{\prime}}\right)_{+}\right)\left(\Psi_{a}\right), \quad F_{a}\left(\overline{\mathscr{M}}_{a}, \overline{\mathscr{L}}_{a}\right)\left(\Psi_{a}\right)=\left(\sum_{a^{\prime} \in \mathcal{S}} F_{a^{\prime}}\left(\overline{\mathscr{M}}_{a^{\prime}}, \overline{\mathscr{L}}_{a^{\prime}}\right)_{+}\right)\left(\Psi_{a}\right)
$$

for all $a \in \mathcal{S}$.

Proof. The string equations (44) imply the invariance conditions

$$
\partial_{6} E_{11} W=\partial_{6} E_{11} \bar{W}=0
$$

Now, recalling Proposition 7 we get the desired result.

\section{The dispersionless limit}

We consider here the dispersionless limit of the multi-component 2D Toda hierarchy. For that aim we use the vector wave functions (35) at a given fixed value $n_{0}$ of the discrete variable $n$. Thus, from Theorem 1 the following auxiliary linear system follows

$$
\partial_{j a}\left(\Psi_{b}\right)=\left(\mathscr{L}_{a}^{j}\right)_{+}\left(\Psi_{b}\right)=\left(\overline{\mathscr{L}}_{a}^{j}\right)_{+}\left(\Psi_{b}\right) \quad a \in \mathcal{S}, j=1,2, \ldots
$$


Let us now introduce slow variables by

$$
t_{\mathrm{sl}, j a}=\epsilon t_{j a}, \quad s_{\mathrm{sl}, a}=\epsilon s_{a},
$$

where $\epsilon$ is a small real parameter and $s_{\mathrm{sl}, a}$ are assumed to be continuous variables. For the sake of simplicity, we will henceforth denote by $\left(t_{j a}, s_{a}\right)$ these slow variables. Moreover, we assume that the wave functions have the quasi-classical form

$$
\Psi_{a}=\exp \left(\frac{\mathscr{S}_{a}}{\epsilon}\right), \quad \mathscr{S}_{a}=\mathscr{S}_{a, 0}+\epsilon \mathscr{S}_{a, 1}+\cdots
$$

with

$$
\mathscr{S}_{a}=\mathcal{T}_{a}+\left\{\begin{array}{ll}
\epsilon \varphi_{1,11} z^{-1}+O\left(z^{-2}\right) & a=1, \\
\epsilon \log \varphi_{1, k 1}+O\left(z^{-1}\right) & a=k \neq 1 \\
\epsilon \log \bar{\varphi}_{0, k 1}+O(z) & a=\bar{k} .
\end{array} \quad \mathcal{T}_{a}:= \begin{cases}\left(\epsilon n_{0}+s_{1}\right) \log z+\sum_{j=1}^{\infty} t_{j l} z^{j}, & a=1, \\
\left(\epsilon n_{0}+s_{k}-\epsilon\right) \log z+\sum_{j=1}^{\infty} t_{j k} z^{j}, & a=k \neq 1, \\
\left(\epsilon n_{0}-s_{\bar{k}}\right) \log z+\sum_{j=1}^{\infty} t_{j \bar{k}} z^{-j}, & a=\bar{k} .\end{cases}\right.
$$

From these expressions we deduce that as $\epsilon \rightarrow 0$

$$
\begin{aligned}
\varphi_{1,11} & =O\left(\epsilon^{-1}\right), \\
\log \varphi_{1,1 k} & =O\left(\epsilon^{-1}\right), \\
\log \bar{\varphi}_{0,1 \bar{k}} & =O\left(\epsilon^{-1}\right) .
\end{aligned} \quad k \neq 1,
$$

As a consequence the coefficients in the operators $\mathscr{L}_{a}, \overline{\mathscr{L}}_{a}$ are Taylor series in $\epsilon$ while those of the Orlov-Schulman operators $\mathscr{M}_{a}, \overline{\mathscr{M}}_{a}$ have at most a simple pole in $\epsilon=0$.

We introduce some new variables

$$
\begin{array}{rlrl}
\sigma_{a} & :=s_{a}, \quad a \neq 1, & \bar{\sigma}_{a}:=s_{a}, \quad a \neq \overline{1}, \\
\sigma_{1}:=\sum_{a \in \mathcal{S}} s_{a}, & \bar{\sigma}_{1}:=\sum_{a \in \mathcal{S}} s_{a},
\end{array}
$$

Observe that

$$
\frac{\partial}{\partial \sigma_{a}}=\frac{\partial}{\partial s_{a}}-\frac{\partial}{\partial s_{1}}, \quad \quad \quad \quad \quad \frac{\partial}{\partial \bar{\sigma}_{a}}=\frac{\partial}{\partial s_{a}}-\frac{\partial}{\partial s_{\overline{1}}}, \quad a \neq \overline{1} .
$$

The zero charge condition implies that $\sigma_{1}=\sigma_{\overline{1}}=0$. Then, we define

$$
\partial_{a}:=\left\{\begin{array}{ll}
\frac{\partial}{\partial \sigma_{a}}, & a \neq 1, \\
-\frac{\partial}{\partial \sigma_{a_{0}}}, & a=1,
\end{array} \quad \bar{\partial}_{a}:= \begin{cases}\frac{\partial}{\partial \bar{\sigma}_{a}}, & a \neq \overline{1} \\
-\frac{\partial}{\partial \bar{\sigma}_{a_{0}}}, & a=\overline{1} .\end{cases}\right.
$$

Notice that

Proposition 9. In the limit $\epsilon \rightarrow 0$ we have that

$$
\begin{aligned}
\mathcal{T}_{a}^{j}\left(\exp \left(\mathscr{S}_{b} / \epsilon\right)\right) & =\exp \left(\mathcal{T}_{a}^{j}\left(\mathscr{S}_{b}\right) / \epsilon\right)=\exp \left(j \partial_{a}\left(\mathscr{S}_{b, 0}\right)+O(\epsilon)\right) \exp \left(\mathscr{S}_{b} / \epsilon\right), \\
\overline{\mathcal{T}}_{a}^{j}\left(\exp \left(\mathscr{S}_{b} / \epsilon\right)\right) & =\exp \left(\overline{\mathcal{T}}_{a}^{j}\left(\mathscr{S}_{b}\right) / \epsilon\right)=\exp \left(j \bar{\partial}_{a}\left(\mathscr{S}_{b, 0}\right)+O(\epsilon)\right) \exp \left(\mathscr{S}_{b} / \epsilon\right), \\
\partial_{j a}\left(\exp \left(\mathscr{S}_{b} / \epsilon\right)\right) & =\left(\partial_{j a}\left(\mathscr{S}_{b, 0}\right)+O(\epsilon)\right) \exp \left(\mathscr{S}_{b} / \epsilon\right) .
\end{aligned}
$$

\subsection{Hamilton-Jacobi equations and dispersionless Whitham hierarchies}

As $\epsilon \rightarrow 0$ it follows that

$$
\begin{aligned}
& \left(\mathscr{L}_{a}\right)_{+}^{j}\left(\Psi_{b}\right)=\left(\mathscr{P}_{j a}\left(\mathrm{e}^{\partial_{a} \mathscr{S}_{b, 0}}\right)+O(\epsilon)\right) \Psi_{b} \\
& \left(\overline{\mathscr{L}}_{a}\right)_{+}^{j}\left(\Psi_{b}\right)=\left(\overline{\mathscr{P}}_{j a}\left(\mathrm{e}^{\partial_{a} \mathscr{S}_{b, 0}}\right)+O(\epsilon)\right) \Psi_{b}
\end{aligned}
$$


where $\mathscr{P}_{j a}$ and $\overline{\mathscr{P}}_{j a}$ are polynomials

$$
\begin{array}{ll}
\mathscr{P}_{j 1}(Z)=Z^{j}+P_{j 1, j-1} Z^{j-1}+\cdots+P_{j 1,0}, & \\
\mathscr{P}_{j a}(Z)=P_{j a, j} Z^{j}+\cdots+P_{j a, 1} Z, & a \neq 1 \\
\overline{\mathscr{P}}_{j \overline{1}}(Z)=\bar{P}_{j \overline{1}, j} Z^{j}+\bar{P}_{j \overline{1}, j-1} Z^{j-1}+\cdots+\bar{P}_{j \overline{1}, 1} Z-\left(1-\delta_{1 a_{0}}\right) \sum_{i=1}^{j} \bar{P}_{j \overline{1}, i}, & \\
\overline{\mathscr{P}}_{j a}(Z)=\bar{P}_{j a, j} Z^{j}+\cdots+\bar{P}_{j a, 1} Z-\left(1-\delta_{a 1}\right) \sum_{i=1}^{j} \bar{P}_{j a, i}, & a \neq \overline{1} .
\end{array}
$$

Hence, as $\epsilon \rightarrow 0$ we get from (46) the following Hamilton-Jacobi type equations

Proposition 10. The following equations holds

$$
\begin{aligned}
& \partial_{j a}\left(\mathscr{S}_{b, 0}\right)=\mathscr{P}_{j a}\left(\mathrm{e}^{\partial_{a} \mathscr{S}_{b, 0}}\right), \\
& \partial_{j a}\left(\mathscr{S}_{b, 0}\right)=\overline{\mathscr{P}}_{j a}\left(\mathrm{e}^{\bar{\partial}_{a} \mathscr{S}_{b, 0}}\right) .
\end{aligned}
$$

Next we show how these equations lead tot he two pictures of the Whitham hierarchy described in the Appendix A.

\subsubsection{KP and Toda dispersionless limits from the Hamilton-Jacobi equations}

From the basic equation

$$
\frac{\partial \Psi_{b}}{\partial t_{11}}=\left(\mathscr{L}_{1}\right)_{+}\left(\Psi_{b}\right)
$$

we get the important formula

$$
\partial_{t_{11}}\left(\mathscr{S}_{b, 0}\right)=\mathrm{e}^{\left(\partial_{s_{1}}-\partial_{s_{a}}\right)\left(\mathscr{S}_{b, 0}\right)}+q_{a}, \quad a \neq 1 .
$$

Where $q_{a}$ is an appropriate function defined in terms of derivatives of the leading coefficient of $\varphi_{1,11}$. Observe that a family of equations as (49) only occurs for the time $t_{11}$ and not for the times $t_{1 a}$ with $a \neq 1$. This is a consequence of the fact that we have chosen the first row in the matrix wave functions, and we are dealing with the shifts of type $\mathcal{T}_{a}$.

\section{The KP-picture dispersionless limit}

Definition 1. We introduce the dispersionless Lax functions in the KP picture, $z_{a}=z_{a}(\boldsymbol{s}, \boldsymbol{t})$ by the implicit relations

$$
p=\partial_{x} \mathscr{S}_{a, 0}\left(z_{a}\right), \quad x:=t_{11},
$$

and the corresponding dispersionless Orlov-Schulman functions by

$$
m_{a}:=\left.\frac{\partial \mathscr{S}_{a, 0}}{\partial z}\right|_{z=z_{a}} .
$$

This definition implies

$$
\left.\mathrm{e}^{\partial_{1} \mathscr{S}_{1,0}}\right|_{z=z_{1}}=p-q_{a_{0}},\left.\quad \quad \quad \mathrm{e}^{\partial_{a} \mathscr{S}_{a, 0}}\right|_{z=z_{a}}=\frac{1}{p-q_{a}}, \quad a \neq 1 .
$$

The next Proposition exhibits the asymptotic form of these functions

Proposition 11. The dispersionless Lax and Orlov-Schulman functions satisfy

$$
\begin{aligned}
& z_{a}^{\mathrm{sg} a}=\left\{\begin{array}{lll}
p+\ell_{1,0}+O\left(p^{-1}\right), & p \rightarrow \infty, & a=1, \\
\frac{\ell_{a, 1}}{p-q_{a}}+\ell_{a, 0}+O\left(p-q_{a}\right), & p \rightarrow q_{a} & a \neq 1,
\end{array}\right. \\
& m_{a}=\left(n_{0}+\operatorname{sg}(a) s_{a}\right) z_{a}^{-1}+\sum_{j=1}^{\infty} j t_{j a} z_{a}^{j-1}+z_{a}^{-1} \begin{cases}\sum_{j=1}^{\infty} \mu_{a j}\left(p-q_{a^{\prime}}\right)^{-j}, & a=1, \\
\sum_{j=1}^{\infty} \mu_{a j}\left(p-q_{a}\right)^{j}, & a \neq 1 .\end{cases}
\end{aligned}
$$


Proof. Particular cases of (36) are

$$
\mathscr{M}_{a}\left(\psi_{a}\right)=z \frac{\mathrm{d} \psi_{a}}{\mathrm{~d} z}, \quad \mathscr{L}_{a}\left(\psi_{a}\right)=z^{\mathrm{sg} a} \psi_{a},
$$

which together with (29) and (33) imply

$$
\begin{aligned}
z^{\mathrm{sg} a} & = \begin{cases}\mathrm{e}^{\partial_{l} \mathscr{S}_{1,0}}+\ell_{1,0}+\ell_{1,-1} \mathrm{e}^{-\partial_{l} \mathscr{S}_{1,0}}+\ell_{1,-2} \mathrm{e}^{-2 \partial_{l} \mathscr{S}_{1,0}}+\cdots, & a=1 \\
\ell_{a, 1} \mathrm{e}^{\partial_{a} \mathscr{S}_{a, 0}}+\ell_{a, 0}+\ell_{a,-1} \mathrm{e}^{-\partial_{a} \mathscr{S}_{a, 0}}+\ell_{a,-2} \mathrm{e}^{-2 \partial_{a} \mathscr{S}_{a, 0}}+\cdots, & a \neq 1,\end{cases} \\
\frac{\partial \mathscr{S}_{a, 0}}{\partial z} & =\left(n_{0}+\operatorname{sg}(a) s_{a}\right) z^{-1}+\sum_{j=1}^{\infty} j t_{j a} z^{j-1}+z^{-1} \sum_{j=1}^{\infty} \mu_{a j} \mathrm{e}^{-j \partial_{a} \mathscr{S}_{a, 0}}
\end{aligned}
$$

and the evaluation at $z=z_{a}$ gives the desired result.

Therefore for $a \neq 1$ we have

$$
\begin{array}{ll}
\left.\left(\partial_{a}\left(\mathscr{S}_{b, 0}\right)\right)\right|_{z=z_{b}}=-\log \left(p-q_{a}\right), & a \neq 1 \\
\left.\left(\partial_{j a}\left(\mathscr{S}_{b, 0}\right)\right)\right|_{z=z_{b}}=\mathscr{P}_{j a}\left(\frac{1}{p-q_{a}}\right)=: \Omega_{j a}, & a \neq 1 \\
\left.\left(\partial_{j 1}\left(\mathscr{S}_{b, 0}\right)\right)\right|_{z=z_{b}}=\mathscr{P}_{j 1}\left(p-q_{a^{\prime}}\right)=: \Omega_{j 1}, & j>1
\end{array}
$$

Then we have that

$$
\mathrm{d} \mathscr{S}_{b, 0}=m_{b} \mathrm{~d} z_{b}+p \mathrm{~d} x-\sum_{a \neq 1} \log \left(p-q_{a}\right) \mathrm{d} s_{a}+\sum_{j, a}^{\prime} \Omega_{j a} \mathrm{~d} t_{j a}
$$

where the $\Sigma^{\prime}$ indicates the sum over the set of indexes $(j, a)$ where $j=1,2, \cdots$ and $a \in \mathcal{S}$ excluding the case $j=1$ and $a=l$. Thus the functions $\mathrm{d} \mathscr{S}_{b, 0}$ determine a solution of the zero-genus Whitham hierarchy with $2 N$ punctures in the KP picture (see Appendix A).

The Toda-picture dispersionless limit We again consider equation (49)

$$
\partial_{t_{11}}\left(\mathscr{S}_{b, 0}\right)=\mathrm{e}^{\left(\partial_{s_{1}}-\partial_{s_{a}}\right)\left(\mathscr{S}_{b, 0}\right)}+q_{a}
$$

which implies

$$
\mathrm{e}^{-\partial_{a_{0}} \mathscr{S}_{b, 0}}=\mathrm{e}^{-\partial_{a} \mathscr{S}_{b, 0}}+Q_{a}, \quad \quad \quad a, a_{0} \neq 1, \quad Q_{a}:=q_{a}-q_{a_{0}}
$$

Definition 2. In the Toda representation the dispersionless Lax function $z_{a}=z_{a}(\boldsymbol{s}, \boldsymbol{t})$ is given by the implicit relation

$$
p=\left.\mathrm{e}^{-\partial_{x} \mathscr{S}_{b, 0}}\right|_{z=z_{b}}, \quad x:=-\sigma_{a_{0}},
$$

and the dispersionless Orlov-Schulman function by

$$
m_{a}:=\left.z \frac{\partial \mathscr{S}_{a, 0}}{\partial z}\right|_{z=z_{a}}
$$

Observing that

$$
\mathrm{e}^{\partial_{a} \mathscr{S}_{b, 0}}=\frac{1}{\mathrm{e}^{-\partial_{a_{0}} \mathscr{S}_{b, 0}-Q_{a}}}
$$

we conclude

$$
\left.\mathrm{e}^{\partial_{x} \mathscr{S}_{1,0}}\right|_{z=z_{1}}=p,\left.\quad \mathrm{e}^{\partial_{a_{0}} \mathscr{S}_{a_{0}, 0}}\right|_{z=z_{a^{\prime}}}=p^{-1},\left.\quad \mathrm{e}^{\partial_{a} \mathscr{S}_{a, 0}}\right|_{z=z_{a}}=\frac{1}{p-Q_{a}}, \quad a_{0} \neq 1 \quad a \neq 1, a_{0} .
$$

Hence, we deduce 
Proposition 12. The dispersionless Lax and Orlov-Schulman functions in the Toda-picture dispersionless limit satisfy

$$
\begin{aligned}
& z_{a}^{\mathrm{sg} a}=\left\{\begin{array}{lll}
p+\ell_{1,0}+O\left(p^{-1}\right), & p \rightarrow \infty, & a=1, \\
\ell_{2,1} p^{-1}+O(1), & p \rightarrow 0, & a=a_{0}, \\
\frac{\ell_{a, 1}}{p-Q_{a}}+O(1), & p \rightarrow Q_{a}, & a \neq 1, a_{0} .
\end{array}\right. \\
& m_{a}=\left(n_{0}+\operatorname{sg}(a) s_{a}\right)+\sum_{j=1}^{\infty} j t_{j a} z_{a}^{j}+ \begin{cases}\sum_{j=1}^{\infty} \mu_{1 j} p^{-j}, & a=1, \\
\sum_{j=1}^{\infty} \mu_{a j} p^{j}, & a=a_{0}, \\
\sum_{j=1}^{\infty} \mu_{a j}\left(p-Q_{a}\right)^{j}, & a \neq 1, a_{0} .\end{cases}
\end{aligned}
$$

Proof. Proceed as in the proof of Proposition 11 and use (50).

As in the KP case we get now

$$
\begin{array}{rlr}
\left.\left(\partial_{a}\left(\mathscr{S}_{b, 0}\right)\right)\right|_{z=z_{b}} & =-\log \left(p-Q_{a}\right), & a \neq 1, a_{0}, \\
\left.\left(\partial_{j a}\left(\mathscr{S}_{b, 0}\right)\right)\right|_{z=z_{b}} & =\mathscr{P}_{j a}\left(\frac{1}{p-Q_{a}}\right)=: \Omega_{j a}, & a \neq 1, a_{0}, \\
\left.\left(\partial_{j 1}\left(\mathscr{S}_{b, 0}\right)\right)\right|_{z=z_{b}} & =\mathscr{P}_{j 1}(p)=: \Omega_{j 1}, & \\
\left.\left(\partial_{j a_{0}}\left(\mathscr{S}_{b, 0}\right)\right)\right|_{z=z_{b}} & =\mathscr{P}_{j a_{0}}\left(p^{-1}\right)=: \Omega_{j a_{0}} . &
\end{array}
$$

Hence we have that

$$
\mathrm{d} \mathscr{S}_{b, 0}=m_{b} \mathrm{~d} \log z_{b}+\log p \mathrm{~d} x-\sum_{a \neq 1, a^{\prime}} \log \left(p-Q_{a}\right) \mathrm{d} s_{a}+\sum_{j \geq 1, a \in \mathcal{S}} \Omega_{j a} \mathrm{~d} t_{j a},
$$

and therefore the functions $\mathscr{S}_{b, 0}$ determine a solution of the zero-genus Whitham hierarchy with $2 N$ punctures in the Toda picture (see Appendix A).

An alternative Toda-picture dispersionless limit From the basic equation

$$
\frac{\partial \Psi_{b}}{\partial t_{1 \overline{1}}}=\left(\overline{\mathscr{L}}_{\overline{1}}\right)_{+}\left(\Psi_{b}\right)
$$

we deduce

$$
\partial_{1 \overline{1}} \mathscr{S}_{b, 0}=r_{1} \mathrm{e}^{\left(\partial_{s_{\overline{1}}}-\partial_{s_{1}}\right)\left(\mathscr{S}_{b, 0}\right)}=r_{a}\left(\mathrm{e}^{\left(\partial_{s_{\overline{1}}}-\partial_{s_{a}}\right)\left(\mathscr{S}_{b, 0}\right)}-1\right), \quad a \neq 1, \overline{1}
$$

for some functions $r_{a}$. Hence,

$$
\mathrm{e}^{\bar{\partial}_{a} \mathscr{S}_{b, 0}}=\frac{\mathrm{e}^{\bar{\partial}_{1} \mathscr{S}_{b, 0}}}{\mathrm{e}^{\bar{\partial}_{1} \mathscr{S}_{b, 0}}+\rho_{a}}, \quad \quad \rho_{a}:=\frac{r_{a}}{r_{1}} .
$$

Now, we take $a_{0}=1$ and define

Definition 3. The dispersionless Lax functions $z_{a}$ are defined by the implicit relation

$$
\left.\partial_{x} \mathscr{S}_{a, 0}\right|_{z=z_{a}}=\log p, \quad x=-\bar{\sigma}_{1}
$$

while the dispersionless Orlov-Schulman functions are defined by

$$
m_{a}:=\left.z_{a} \frac{\partial \mathscr{S}_{a, 0}}{\partial z}\right|_{z=z_{a}}
$$

Observe that

$$
\left.\mathrm{e}^{\partial_{1} \mathscr{S}_{1,0}}\right|_{z=z_{1}}=p,\left.\quad \quad \mathrm{e}^{\partial_{\overline{1}} \mathscr{S}_{\overline{1}, 0}}\right|_{z=z_{\overline{1}}}=p^{-1},\left.\quad \mathrm{e}^{\partial_{a} \mathscr{S}_{a, 0}}\right|_{z=z_{a}}=\frac{p}{p+\rho_{a}}, \quad a \neq 1, \overline{1} .
$$


Proposition 13. The dispersionless Lax and Orlov-Schulman functions are of the following form

$$
\begin{aligned}
& z_{a}^{\mathrm{sg} a}=\left\{\begin{array}{lll}
p+\bar{\ell}_{1,0}+O\left(p^{-1}\right), & p \rightarrow \infty, & a=1 \\
\bar{\ell}_{\overline{1}, 1} p^{-1}+\bar{\ell}_{\overline{1}, 0}+O(p), & p \rightarrow 0, & a=\overline{1} \\
\bar{\ell}_{a, 1} \frac{p}{p+\rho_{a}}+\bar{\ell}_{a, 0}+O\left(\left(\frac{p}{p+\rho_{a}}\right)^{-1}\right), & p \rightarrow-\rho_{a}, \quad a \neq 1,
\end{array}\right. \\
& m_{a}=\left(n_{0}+\operatorname{sg}(a) s_{a}\right)+\sum_{j=1}^{\infty} j t_{j a} \bar{z}_{a}^{j-1}+ \begin{cases}\sum_{j=1}^{\infty} \mu_{a j} p^{-j}, & a=1, \\
\sum_{j=1}^{\infty} \mu_{a j} p^{j}, & a=\overline{1}, \\
\sum_{j=1}^{\infty} \mu_{a j}\left(\frac{p}{p+\rho_{a}}\right)^{j}, & a \neq 1, \overline{1} .\end{cases}
\end{aligned}
$$

Proof. Particular cases of (36) are

$$
\overline{\mathscr{M}}_{a}\left(\Psi_{a}\right)=z \frac{\mathrm{d} \Psi_{a}}{\mathrm{~d} z}, \quad \overline{\mathscr{L}}_{a}\left(\Psi_{a}\right)=z^{\mathrm{sg} a} \Psi_{a}
$$

which together with (29) and (33) imply

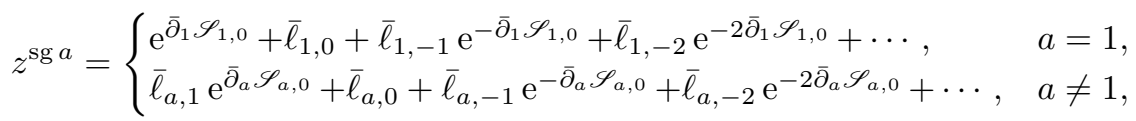

$$
\begin{aligned}
& z \frac{\partial \mathscr{S}_{a, 0}}{\partial z}=\left(n_{0}+\operatorname{sg}(a) s_{a}\right)+\sum_{j=1}^{\infty} j t_{j a} z^{j}+\sum_{j=1}^{\infty} \bar{\mu}_{a j} \mathrm{e}^{-j \bar{\partial}_{a} \mathscr{S}_{a, 0}} .
\end{aligned}
$$

and the evaluation of these expressions at $z=z_{a}$ gives the result.

Therefore,

$$
\begin{array}{rlrl}
\left.\partial_{x}\left(\mathscr{S}_{b, 0}\right)\right|_{z=z_{b}} & =\log p, & \\
\left.\partial_{a}\left(\mathscr{S}_{b, 0}\right)\right|_{z=z_{b}} & =\log \left(\frac{p}{p+\rho_{a}}\right), & & a \neq 1, \overline{1}, \\
\left.\left(\partial_{j a}\left(\mathscr{S}_{b, 0}\right)\right)\right|_{z=z_{b}} & =\overline{\mathscr{P}}_{j a}\left(\frac{p}{p+\rho_{a}}\right)=: \Omega_{j a}, & & a \neq 1, \overline{1}, \\
\left.\partial_{j \overline{1}}\left(\mathscr{S}_{b, 0}\right)\right|_{z=z_{b}} & =\overline{\mathscr{P}}_{j \overline{1}}\left(p^{-1}\right)=: \Omega_{j \overline{1}}, & \\
\left.\left(\partial_{j 1}\left(\mathscr{S}_{b, 0}\right)\right)\right|_{z=z_{b}} & =\overline{\mathscr{P}}_{j 1}(p)=: \Omega_{j 1}, & j>1 .
\end{array}
$$

In this way we have

$$
\mathrm{d} \mathscr{S}_{b, 0}=m_{b} \mathrm{~d} \log z_{b}+\log (p) \mathrm{d} x+\sum_{a \neq \overline{1}, 1} \log \left(\frac{p}{p+\rho_{a}}\right) \mathrm{d} s_{a}+\sum_{j \geq 1, a \in \mathcal{S}} \Omega_{j a} \mathrm{~d} t_{j a} .
$$

As we will show at the end of this section the functions $\mathscr{S}_{b, 0}$ determine a solution of the zero-genus Whitham hierarchy with $2 N$ punctures in the Toda picture.

\subsection{The dispersionless limits of the string equations}

Let us consider operators of the form

$$
F_{a}\left(\mathscr{M}_{a}, \mathscr{L}_{a}\right)=\sum_{i \geq 0, j \in \mathbb{Z}} F_{a i j} \mathscr{M}_{a}^{i} \mathscr{L}_{a}^{j}, \quad F_{a}\left(\overline{\mathscr{M}}_{a}, \overline{\mathscr{L}}_{a}\right)=\sum_{i \geq 0, j \in \mathbb{Z}} F_{a i j} \overline{\mathscr{M}}_{a}^{i} \overline{\mathscr{L}}_{a}^{j}
$$

In order to formulate their dispersionless limits it is convenient to assume that the coefficients satisfy as $\epsilon \rightarrow 0$ that

$$
F_{a i j}=F_{a i j, 0} \epsilon^{i}+O\left(\epsilon^{i+1}\right) .
$$


Recalling (36) and observing that

$$
\begin{aligned}
\left(z \frac{\mathrm{d}}{\mathrm{d} z}\right)^{i} & =z^{i} \frac{\mathrm{d}^{i}}{\mathrm{~d} z^{i}}+\sum_{i^{\prime}=2}^{i}\left(\begin{array}{c}
i^{\prime} \\
2
\end{array}\right) z^{i^{\prime}-1} \frac{\mathrm{d}^{i^{\prime}-1}}{\mathrm{~d} z^{i^{\prime}-1}} \\
\frac{\partial^{i} \Psi_{a}}{\partial z^{i}} & =\left(\epsilon^{-i}\left(\frac{\partial \mathscr{S}_{a, 0}}{\partial z}\right)^{i}+O\left(\epsilon^{-i+1}\right)\right) \Psi_{a}
\end{aligned}
$$

we get

$$
\left(\Psi_{a} \overleftarrow{F_{a}\left(z \frac{\mathrm{d}}{\mathrm{d} z}, z^{\mathrm{sg} a}\right)}=\left[\sum_{i \geq 0, j \in \mathbb{Z}} F_{a i j, 0} z^{\operatorname{sg}(a) j}\left(\frac{\partial \mathscr{S}_{a, 0}}{\partial z}\right)^{i}+O(\epsilon)\right] \Psi_{a}\right.
$$

Hence,

$$
F_{a, 0}\left(z_{a}, m_{a}\right):=\left[\lim _{\epsilon \rightarrow 0}\left(\Psi_{a}\right) \overleftarrow{F_{a}\left(z \frac{\mathrm{d}}{\mathrm{d} z}, z^{\mathrm{sg} a}\right)} \Psi_{a}^{-1}\right]_{z=z_{a}}= \begin{cases}\sum_{i \geq 0, j \in \mathbb{Z}} F_{a i j, 0} z_{a}^{i+\operatorname{sg}(a) j} m_{a}^{i}, & \mathrm{KP} \\ \sum_{i \geq 0, j \in \mathbb{Z}} F_{a i j, 0} z_{a}^{\mathrm{sg}(a) j} m_{a}^{i}, & \text { Toda. }\end{cases}
$$

We define

$$
F_{a, 0+}:= \begin{cases}{\left[\lim _{\epsilon \rightarrow 0} \frac{F_{a}\left(\mathscr{M}_{a}, \mathscr{L}_{a}\right)_{+}\left(\Psi_{a}\right)}{\Psi_{a}}\right]_{z=z_{a}},} & \text { unbared cases } \\ {\left[\lim _{\epsilon \rightarrow 0} \frac{F_{a}\left(\overline{\mathscr{M}}_{a}, \overline{\mathscr{L}}_{a}\right)_{+}\left(\Psi_{a}\right)}{\Psi_{a}}\right]_{z=z_{a}},} & \text { bared cases }\end{cases}
$$

Proposition 14. Given

$$
F_{a}\left(\mathscr{M}_{a}, \mathscr{L}_{a}\right)=\sum_{j \in \mathbb{Z}} f_{a j} \mathcal{T}_{a}^{j}, \quad \quad \text { or } \quad F_{a}\left(\overline{\mathscr{M}}_{a}, \overline{\mathscr{L}}_{a}\right)=\sum_{j \in \mathbb{Z}} \bar{f}_{a j} \overline{\mathcal{T}}_{a}^{j},
$$

with $f_{a i}=f_{a i \mid 0}+O(\epsilon)$ as $\epsilon \rightarrow 0$, their dispersionless limits are

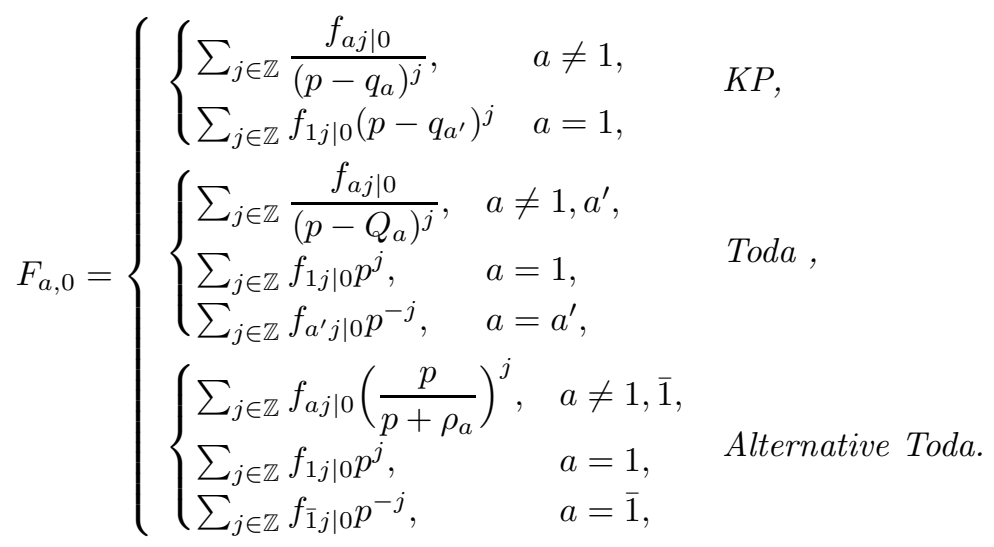

Moreover,

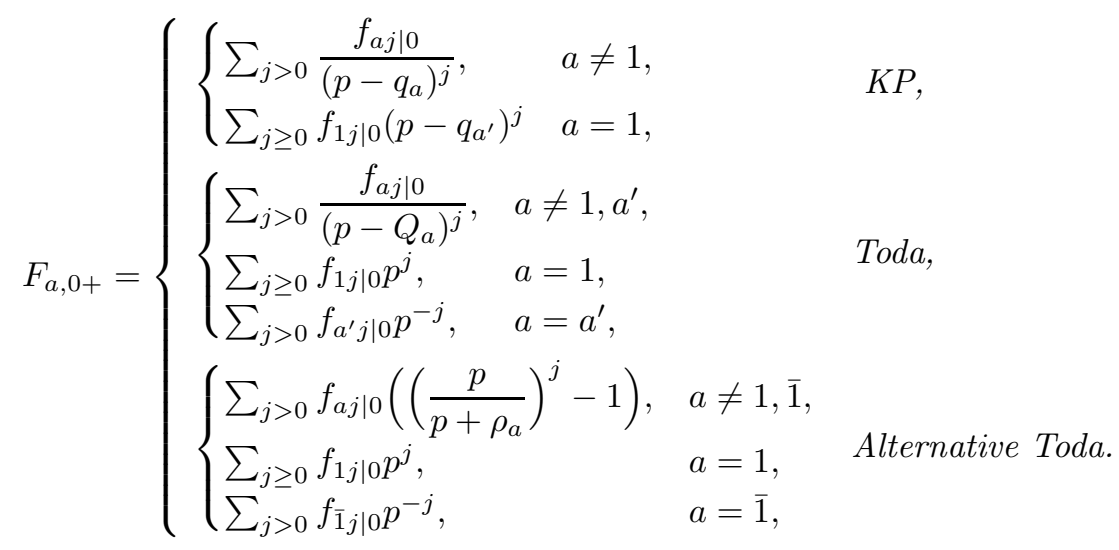


In particular

$$
\Omega_{j a}= \begin{cases}\left(z_{a}^{\mathrm{gg}(a) j}\right)_{+}, & \text {KP, Toda }, \\ \left(\bar{z}_{a}^{\mathrm{gg}(a) j}\right)_{+}, & \text {Alternative Toda } .\end{cases}
$$

Proof. The formulae follow from the identity

$$
\begin{aligned}
\left(\Psi_{a}\right) \overleftarrow{F_{a}\left(z \frac{\mathrm{d}}{\mathrm{d} z}, z^{\mathrm{sg} a}\right)} & =F_{a}\left(\mathscr{M}_{a}, \mathscr{L}_{a}\right)\left(\Psi_{a}\right)=\sum_{j \in \mathbb{Z}} f_{a j} \mathrm{e}^{j \partial_{a} \mathscr{S}_{a, 0}+O(\epsilon)} \Psi_{a} \\
& =F_{a}\left(\overline{\mathscr{M}}_{a}, \overline{\mathscr{L}}_{a}\right)\left(\Psi_{a}\right)=\sum_{j \in \mathbb{Z}} f_{a j} \mathrm{e}^{j \bar{\partial}_{a} \mathscr{S}_{a, 0}+O(\epsilon)} \Psi_{a} .
\end{aligned}
$$

As a consequence

Proposition 15. If the string equations (44) hold, their corresponding dispersionless limits

$$
F_{a, 0}\left(z_{a}, m_{a}\right)=\sum_{b \in \mathcal{S}} F_{b, 0+}, \quad \forall a \in \mathcal{S}
$$

are satisfied.

Proof. It follows from Proposition 8

These dispersionless string equations are of the type considered in 8 . for the dispersionless Whitham hierarchy. Moreover, given a decomposition $\mathcal{S}=\mathcal{I} \cup \mathcal{J}$ into two disjoint subsets, we may take

$$
P_{a, 0}=\left\{\begin{array}{ll}
z_{a}^{\ell_{a}}, & a \in \mathcal{I}, \\
-\frac{m_{a}}{\ell_{a} z^{\ell_{a}-1}}, & a \in \mathcal{J},
\end{array} \quad Q_{a, 0}= \begin{cases}\frac{m_{a}}{\ell_{a} z^{\ell_{a}-1}}, & a \in \mathcal{I}, \\
z_{a}^{\ell_{a}}, & a \in \mathcal{J} .\end{cases}\right.
$$

The corresponding dispersive string equations are

$$
\begin{aligned}
& E_{11}\left(\sum_{k \in(\mathcal{I} \cap \mathbb{S})} L^{\ell_{k}} C_{k k}-\sum_{k \in(\mathcal{J} \cap \mathbb{S})} \ell_{k}^{-1} M L^{-\ell_{k}+1} C_{k k}\right)=E_{11}\left(\sum_{k \in(\mathcal{I} \cap \overline{\mathbb{S}})} \bar{L}^{-\ell_{k}} \bar{C}_{k k}-\sum_{k \in(\mathcal{J} \cap \overline{\mathbb{S}})} \ell_{k}^{-1} \bar{M} \bar{L}^{\ell_{k}-1} \bar{C}_{k k}\right), \\
& E_{11}\left(\sum_{k \in(\mathcal{I} \cap \mathbb{S})} \ell_{k}^{-1} M L^{-\ell_{k}+1} C_{k k}+\sum_{k \in(\mathcal{J} \cap \mathbb{S})} L^{\ell_{k}} C_{k k}\right)=E_{11}\left(\sum_{k \in(\mathcal{I} \cap \overline{\mathbb{S}})} \ell_{k}^{-1} \bar{M} \bar{L}^{\ell_{k}-1} \bar{C}_{k k}+\sum_{k \in(\mathcal{J} \cap \bar{S})} \bar{L}^{-\ell_{k}} \bar{C}_{k k}\right) .
\end{aligned}
$$

If $\mathcal{J}=\varnothing$ we get

$$
\begin{aligned}
E_{11} \sum_{k=1}^{N} L^{\ell_{k}} C_{k k} & =E_{11} \sum_{k=1}^{N} \bar{L}^{-\ell_{k}} \bar{C}_{k k}, \\
E_{11} \sum_{k=1}^{N} \ell_{k}^{-1} M L^{-\ell_{k}+1} C_{k k} & =E_{11} \sum_{k=1}^{N} \ell_{k}^{-1} \bar{M} \bar{L}^{\ell_{k}-1} \bar{C}_{k k} .
\end{aligned}
$$

For positive integers $\ell_{a}$ the dispersionless limits of these dispersive string equations describe the algebraic orbits of the genus 0 Whitham hierarchy [24]. The first of these dispersive string equations gives the multigraded reduction as discussed in [1].

We return to the equivalence of the alternative Toda and Toda pictures. First we notice that within alternative Toda picture we have Laurent expansions in $Z_{a}$

$$
Z_{a}:=\frac{p}{p+\rho_{a}}=1-\rho_{a} \zeta_{a}, \quad \zeta_{a}:=\frac{1}{p+\rho_{a}} .
$$

The functions $Z_{a}^{j}$ are singular at $p=-\rho_{a}$ and $\lim _{p \rightarrow \infty} Z_{a}^{j}=1$, thus the linear combinations of factors $Z_{a}^{j}-1$ which appear in the construction of the $\Omega_{j a}$, lead to singular functions in $p=-\rho_{a}$ normalized to 0 at infinity. Hence, if we express $z_{a}$ as Laurent series in $\zeta_{a}$, the function $\Omega_{j a}$ is just the singular part corresponding to the 
projection to power series in $\zeta_{a}$ with non constant term. Thus, we recover the Toda picture of the genus 0 Whitham hierarchy (see Appendix A).

Given operators $P_{a}$ and $Q_{a}$ as in Proposition 3 we get for the commutator

$$
\begin{aligned}
\left(\Psi_{a}\right) \overleftarrow{\left.P_{a}, Q_{a}\right]}\left(z \frac{\mathrm{d}}{\mathrm{d} z}, z^{\operatorname{sg} a}\right)=\left(\Psi_{a}\right)\left[\overleftarrow{P_{a}\left(z \frac{\mathrm{d}}{\mathrm{d} z}, z^{\operatorname{sg} a}\right)}, \overleftarrow{Q_{a}\left(z \frac{\mathrm{d}}{\mathrm{d} z}, z^{\operatorname{sg} a}\right)}\right] \\
=\left[\sum_{\substack{i_{1}, i_{2} \geq 0 \\
j_{1}, j_{2} \in \mathbb{Z}}} P_{a i_{1} j_{1}, 0} Q_{a i_{2} j_{2}, 0} \operatorname{sg}(a)\left(i_{2} j_{1}-i_{1} j_{2}\right) z^{\operatorname{sg}(a)\left(j_{1}+j_{2}\right)+i_{1}+i_{2}-1}\left(\frac{\partial \mathscr{S}_{a, 0}}{\partial}\right)^{i_{1}+i_{2}-1} \epsilon+O\left(\epsilon^{2}\right)\right] \Psi_{a}
\end{aligned}
$$

so that for the KP picture we find

$$
\begin{aligned}
{\left[\lim _{\epsilon \rightarrow 0}\left(\Psi_{a}\right) \overleftarrow{\epsilon^{-1}\left[P_{a}, Q_{a}\right]}\left(z \frac{\mathrm{d}}{\mathrm{d} z}, z\right) \Psi_{a}^{-1}\right]_{z=z_{a}} } & =\sum_{\substack{i_{1}, i_{2} \geq 0 \\
j_{1}, j_{2} \in \mathbb{Z}}} P_{a i_{1} j_{1}, 0} Q_{a i_{2} j_{2}, 0} \operatorname{sg}(a)\left(i_{2} j_{1}-i_{1} j_{2}\right) z_{a}^{\mathrm{sg}(a)\left(j_{1}+j_{2}\right)+i_{1}+i_{2}-1} m_{a}^{i_{1}+i_{2}-1} \\
& =\left\{P_{0, a}, Q_{0, a}\right\}_{0}
\end{aligned}
$$

while for the Toda picture we have

$$
\begin{aligned}
{\left[\lim _{\epsilon \rightarrow 0}\left(\Psi_{a}\right) \overleftarrow{\epsilon^{-1}\left[P_{a}, Q_{a}\right]}\left(z \frac{\mathrm{d}}{\mathrm{d} z}, z\right) \Psi_{a}^{-1}\right]_{z=z_{a}} } & =\sum_{\substack{i_{1}, i_{2} \geq 0 \\
j_{1}, j_{2} \in \mathbb{Z}}} P_{a i_{1} j_{1}, 0} Q_{a i_{1} j_{1}, 0} \operatorname{sg}(a)\left(i_{2} j_{1}-i_{1} j_{2}\right) z_{a}^{\mathrm{sg}(a)\left(j_{1}+j_{2}\right)} m_{a}^{i_{1}+i_{2}-1} \\
& =\left\{P_{0, a}, Q_{0, a}\right\}_{1} .
\end{aligned}
$$

Thus,

$$
\left[P_{a}, Q_{a}\right]=\epsilon \quad \Longrightarrow \quad\left\{P_{a, 0}, Q_{a, 0}\right\}_{0}=1 \text { or }\left\{P_{a, 0}, Q_{a, 0}\right\}_{1}=1
$$

\section{Appendix A: Whitham hierarchies in the zero genus case}

The zero-genus Whitham hierarchies [24] are systems of flows on a phase space $\widehat{M}_{0}$ of data associated to algebraic Riemann surfaces of genus 0 . The points of $\widehat{M}_{0}$ are given $\left(\Gamma, Q_{a}, z_{a}^{-1}\right)$, where $\Gamma$ is an algebraic Riemann surface of genus $0, Q_{a}$ are $N$ points (punctures) of $\Gamma$ and $z_{a}^{-1}$ denote local coordinates around each $Q_{a}$ such that $z_{a}^{-1}\left(Q_{a}\right)=0$. In order to formulate Whitham flows on $\widehat{M}_{0}$ it is convenient to introduce a meromorphic function $p=p(Q)$ on $\Gamma$ such that the local coordinates have asymptotic expansions of the form

$$
z_{a}= \begin{cases}p+\ell_{1,0}+\sum_{n=1}^{\infty} \frac{\ell_{1,-n}}{p^{n}}, & a=1, \\ \frac{\ell_{a, 1}}{p-q_{a}}+\sum_{n=0}^{\infty} \ell_{a,-n}\left(p-q_{a}\right)^{n}, & a=2, \ldots, N,\end{cases}
$$

where $p\left(Q_{a}\right)=q_{a}$ with $q_{1}=\infty$. In general the points of the phase space $\widehat{M}_{0}$ are characterized by an infinite number of parameters $\boldsymbol{w}:=\left(w_{i}\right)$ of the set $\left(q_{a}, \ell_{a, n}\right)$. However, under appropriate reduction conditions on the form of $\Gamma$ only a finite number of these parameters are independent and constitute a coordinate system for $\widehat{M}_{0}$.

\section{Example: Algebraic orbits}

If we restrict to zero-genus Riemann surfaces $\Gamma$ of the form

$$
\lambda=p^{n_{1}}+\sum_{n=0}^{n_{1}-1} u_{1 n} p^{n}+\sum_{i=2}^{N} \sum_{n=1}^{n_{q}} \frac{u_{i n}}{\left(p-q_{i}\right)^{n}},
$$


we may take $Q_{a}=\left(\lambda_{a}, p_{a}\right)=\left(\infty, q_{a}\right)(a=1, \ldots, q)$, with corresponding local coordinates given by

$$
z_{a}=\lambda^{1 / n_{a}} .
$$

The function $p(\lambda, p)=p$ is meromorphic on $\Gamma$ and the local coordinates have asymptotic expansions of the form (52). In this case the points of the phase space $\widehat{M}_{0}$ are characterized by the parameters $\boldsymbol{w}=$ $\left(\left\{q_{a}\right\}_{a=2}^{N},\left\{u_{1 n}\right\}_{n=0}^{n_{1}}, \ldots,\left\{u_{N n}\right\}_{N=1}^{n_{N}}\right)$

The Whitham flows $\boldsymbol{w}(\boldsymbol{t})=\left(w_{i}(\boldsymbol{t})\right)$ are introduced through sets $\boldsymbol{\Omega}:=\left\{\Omega_{A}(\boldsymbol{w}, p)\right\}$ of functions, with meromorphic differentials $\partial_{p} \Omega_{A}(\boldsymbol{w}, p) \mathrm{d} p$, which satisfy the conditions:

1. One of the functions $\Omega_{A_{0}}$ is independent of the data $\boldsymbol{w}$.

2. There exist local functions $S_{a}\left(\boldsymbol{t}, z_{a}\right)$ around the punctures satisfying

$$
\partial_{A} S_{a}\left(\boldsymbol{t}, z_{a}\right)=\Omega_{A}\left(\boldsymbol{w}(\boldsymbol{t}), p\left(\boldsymbol{t}, z_{a}\right)\right) .
$$

Here $\partial_{A}:=\partial / \partial t_{A}$ and $\boldsymbol{t}$ denotes the set of flow parameters $t_{A}$.

The first condition only demands to include a function of the form $\Omega(p)$ in $\boldsymbol{\Omega}$. On the other hand, it is obvious that the second condition is satisfied if and only if the following Zakharov-Shabat equations are satisfied

$$
\partial_{B} \Omega_{A}-\partial_{A} \Omega_{B}+\left\{\Omega_{A}, \Omega_{B}\right\}=0,
$$

where $\partial_{t}:=\sum_{i} \partial_{t} w_{i} \partial_{w_{i}}$ for $t=t_{A}, t_{B}$, and $\{$,$\} denotes the Poisson bracket$

$$
\{F, G\}:=\omega(p)\left(\partial_{p} F \partial_{x} G-\partial_{x} F \partial_{p} G\right), \quad \omega(p):=\left(\partial_{p} \Omega_{A_{0}}(p)\right)^{-1}, \quad x:=t_{A_{0}} .
$$

We may write (54) as

$$
\mathrm{d} S_{a}=m_{a} \mathrm{~d} \Omega_{A_{0}}\left(z_{a}\right)+\sum_{A} \Omega_{A} \mathrm{~d} t_{A}, \quad m_{a}:=\omega\left(z_{a}\right)^{-1} \frac{\partial S_{a}}{\partial z_{a}},
$$

which implies

$$
\mathrm{d} \Omega_{A_{0}}\left(z_{a}\right) \wedge \mathrm{d} m_{a}=\sum_{A} \mathrm{~d} \Omega_{A} \wedge \mathrm{d} t_{A},
$$

and by equating the coefficients of $\mathrm{d} p \wedge \mathrm{d} x$ in both members of (57) yields

$$
\left\{z_{a}, m_{a}\right\}=\omega\left(z_{a}\right) .
$$

Moreover, if we identify the coefficients of $\mathrm{d} p \wedge \mathrm{d} t_{A}$ and $\mathrm{d} x \wedge \mathrm{d} t_{A}$ in (57) we get

$$
\left\{\begin{array}{l}
\partial_{p} z_{a} \partial_{A} m_{a}-\partial_{A} z_{a} \partial_{p} m_{a}=\omega\left(z_{a}\right) \partial_{p} \Omega_{A}, \\
\partial_{x} z_{a} \partial_{A} m_{a}-\partial_{A} z_{a} \partial_{x} m_{a}=\omega\left(z_{a}\right) \partial_{x} \Omega_{A},
\end{array}\right.
$$

so that taking (58) into account we deduce the system of Lax equations

$$
\partial_{A} z_{a}=\left\{\Omega_{A}, z_{a}\right\}, \quad \partial_{A} m_{a}=\left\{\Omega_{A}, m_{a}\right\} .
$$

As it was shown in [?]-[27 important classes of solutions of the zero-genus Whitham hierarchy can be obtained from systems of canonical pairs of constrains (string equations) of the form

$$
\left\{\begin{array}{l}
P_{1}\left(z_{1}, m_{1}\right)=P_{2}\left(z_{2}, m_{2}\right)=\cdots=P_{N}\left(z_{N}, m_{N}\right), \\
Q_{1}\left(z_{1}, m_{1}\right)=Q_{2}\left(z_{2}, m_{2}\right)=\cdots=Q_{N}\left(z_{N}, m_{N}\right),
\end{array}\right.
$$

where $\left(P_{a}, Q_{a}\right)$ are $N$ pairs of canonically conjugate functions

$$
\left\{P_{a}(p, x), Q_{a}(p, q)\right\}=\omega(p) .
$$

In particular this type of methods applies for finding solutions for algebraic orbits. Indeed these solutions are associated to string equations generated by

$$
P_{a}(p, x)=p^{n_{a}}, \quad Q_{a}(p, x)=\frac{x}{n_{a} p^{n_{a}-1}}+f_{a}(p) .
$$




\section{The KP picture}

The KP picture of the zero-genus Whitham hierarchy with $N$ punctures 24 is formulated by assuming $\ell_{1,0} \equiv 0$ in the asymptotic expansions (52) and by taking the following functions $\Omega_{A}$

$$
\Omega_{n a}:= \begin{cases}\left(z_{a}^{n}\right)_{(a,+)}, & n \geq 1, \\ -\log \left(p-q_{a}\right), & n=0, \quad a=2, \ldots, N .\end{cases}
$$

Here $(\cdot)_{(a,+)}$ stand for the projectors on the subspaces generated by $\left\{p^{n}\right\}_{n=0}^{\infty}($ case $a=1)$ and $\left\{\left(p-q_{a}\right)^{-n}\right\}_{n=1}^{\infty}$ (cases $a \geq 2$ ). In this case

$$
A_{0}=(1,1), \quad x=t_{1,1}, \quad \Omega_{A_{0}}=p,
$$

and the Poisson bracket is given by

$$
\{F, G\}:=\partial_{p} F \partial_{x} G-\partial_{x} F \partial_{p} G .
$$

The functions $\Omega_{A}$ satisfy the compatibility conditions (55) so that there exist functions $S_{a}$ such that

$$
\mathrm{d} S_{a}=m_{a} \mathrm{~d} z_{a}+p \mathrm{~d} x-\sum_{a \neq 1} \log \left(p-q_{a}\right) \mathrm{d} t_{0 a}+\sum_{a=1}^{N} \sum_{n \geq 1} \Omega_{n a} \mathrm{~d} t_{n a} .
$$

\section{The Toda picture}

A simple redefinition of the meromorphic function $p(Q)$ used to define the KP flows of the Whitham hierarchy with $N$ punctures supplies a different picture (the Toda picture) of the hierarchy. Indeed if we set

$$
p_{\text {Toda }}=p_{\mathrm{KP}}-q_{a_{0}}
$$

for a given index $a_{0}$, then now $u_{1,1}=q_{a_{0}}$ and we may take

$$
A_{0}:=\left(0, a_{0}\right), \quad x:=-t_{0, a_{0}}, \quad \Omega_{A_{0}}:=-\log p .
$$

Thus the Poisson bracket is given by

$$
\{F, G\}:=p\left(\partial_{p} F \partial_{x} G-\partial_{x} F \partial_{p} G\right),
$$

and the functions $S_{a}$ satisfy

$$
\mathrm{d} S_{a}=m_{a} \mathrm{~d} \log z_{a}+\log p \mathrm{~d} x-\sum_{a \neq 1, a_{0}} \log \left(p-q_{a}\right) \mathrm{d} t_{0 a}+\sum_{a=1}^{N} \sum_{n \geq 1} \Omega_{n a} \mathrm{~d} t_{n a} .
$$

\section{Appendix B: Proof of Proposition (6)}

The proof of Proposition (6) requires the following Lemma.

Lemma 1. 1. Given $T=\sum_{j \in \mathbb{Z}} c_{j} \mathcal{T}_{a}^{j} \in \mathfrak{t}_{a}, a \neq 1$, then

$$
\begin{aligned}
& \left\{\begin{array}{l}
T\left(E_{11} W\right)=T_{>}\left(W_{1 k}\right) E_{1 k}+T_{\leq}\left(W_{11}\right) E_{1}+\mathfrak{g}_{-} W_{0}, \\
T\left(E_{11} \bar{W}\right)=\mathfrak{g}_{+} \bar{W}_{0},
\end{array} \quad a=k \neq 1,\right. \\
& \begin{cases}T\left(E_{11} W\right)=T_{\leq}\left(W_{11}\right) E_{11}+\mathfrak{g}_{-} W_{0}, & a=\bar{k}, \\
T\left(E_{11} \bar{W}\right)=T_{>}\left(\bar{W}_{1 k}\right) E_{1 k}+\mathfrak{g}_{+} \bar{W}_{0}, & \end{cases}
\end{aligned}
$$

2. Given $T=\sum_{j \in \mathbb{Z}} c_{j} \mathcal{T}_{l}^{j} \in \mathfrak{t}_{1}$, then

$$
\begin{aligned}
& \left\{\begin{array}{l}
T\left(E_{11} W\right)=T_{\geq}\left(W_{11}\right) E_{11}+T_{<}\left(W_{1 l_{0}}\right) E_{1 l_{0}}+\mathfrak{g}_{-} W_{0}, \\
T\left(E_{11} \bar{W}\right)=\mathfrak{g}_{+} \bar{W}_{0},
\end{array} \quad a_{0}=l_{0} \neq 1,\right. \\
& \begin{cases}T\left(E_{11} W\right)=T_{\geq}\left(W_{11}\right) E_{11}+\mathfrak{g}_{-} W_{0}, & a_{0}=\overline{l_{0}}, \\
T\left(E_{11} \bar{W}\right)=T_{<}\left(\bar{W}_{1 l_{0}}\right) E_{1 l_{0}}+\mathfrak{g}_{+} \bar{W}_{0}, & \end{cases}
\end{aligned}
$$


3. Given $T=\sum_{j \in \mathbb{Z}} c_{j}\left(\overline{\mathcal{T}}_{a}^{j}-1\right)+c_{0} \in \overline{\mathfrak{t}}_{a}, a \neq \overline{1}, 1$, then

$$
\begin{aligned}
& \begin{cases}T\left(E_{11} W\right)=T_{>}\left(W_{1 k}\right) E_{1 k}+c_{0} W_{11} E_{11}+\mathfrak{g}_{-} W_{0}, & a=k \neq 1, \\
T\left(E_{11} \bar{W}\right)=T_{<}\left(\bar{W}_{11}\right) E_{11}+\mathfrak{g}_{+} \bar{W}_{0}, & a=\bar{k} \neq \overline{1},\end{cases} \\
& \begin{cases}T\left(E_{11} W\right)=c_{0} W_{11} E_{11}+\mathfrak{g}_{-} W_{0}, & \\
T\left(E_{11} \bar{W}\right)=T_{>}\left(\bar{W}_{l k}\right) E_{l k}+T_{<}\left(\bar{W}_{11}\right) E_{11}+\mathfrak{g}_{+} \bar{W}_{0}, & \end{cases}
\end{aligned}
$$

4. Given $T=\sum_{j \in \mathbb{Z}} c_{j} \overline{\mathcal{T}}_{1}^{j} \in \overline{\mathfrak{t}}_{1}$, then

$$
\left\{\begin{array}{l}
T\left(E_{11} W\right)=T_{\geq}\left(W_{11}\right) E_{11}+\mathfrak{g}_{-} W_{0}, \\
T\left(E_{11} \bar{W}\right)=T_{<}\left(\bar{W}_{11}\right) E_{11}+\mathfrak{g}_{+} \bar{W}_{0},
\end{array}\right.
$$

5. Given $T=\sum_{j \in \mathbb{Z}} c_{j}\left(\overline{\mathcal{T}}_{\overline{1}}^{j}-1\right)+c_{0} \in \overline{\mathfrak{t}}_{\overline{1}}$, with $a_{0} \neq 1$, then

$$
\begin{aligned}
& \begin{cases}T\left(E_{11} W\right)=T_{<}\left(W_{1 l_{0}}\right) E_{1 l_{0}}+c_{0} W_{l l} E_{11}+\mathfrak{g}_{-} W_{0}, & a_{0}=l_{0} \neq 1, \\
T\left(E_{11} \bar{W}\right)=T_{>}\left(\bar{W}_{11}\right)+\mathfrak{g}_{+} \bar{W}_{0}, & \end{cases} \\
& \begin{cases}T\left(E_{11} W\right)=c_{0} W_{11} E_{11}+\mathfrak{g}_{-} W_{0}, & a_{0}=\bar{l}_{0}, \\
T\left(E_{11} \bar{W}\right)=T_{>}\left(\bar{W}_{11}\right) E_{11}+T_{<}\left(\bar{W}_{1 l_{0}}\right) E_{1 l_{0}}+\mathfrak{g}_{+} \bar{W}_{0}, & \end{cases}
\end{aligned}
$$

6. Given $T=\sum_{j \in \mathbb{Z}} c_{j} \overline{\mathcal{T}}_{\overline{1}}^{j} \in \overline{\mathfrak{t}}_{\overline{1}}$ with $a_{0}=1$, then

$$
\left\{\begin{array}{l}
T\left(E_{11} W\right)=T_{\leq}\left(W_{11}\right) E_{11}+\mathfrak{g}_{-} W_{0}, \\
T\left(E_{11} \bar{W}\right)=T_{>}\left(\bar{W}_{11}\right) E_{11}+\mathfrak{g}_{+} \bar{W}_{0} .
\end{array}\right.
$$

Proof. We only prove 1) since the others relations are proven similarly. From (66) observe that

$$
\begin{aligned}
\mathcal{T}_{k}^{j}\left(E_{11} W\right) & =\mathcal{T}_{k}^{j}\left(E_{11} S\right) \mathcal{T}_{k}^{j}\left(W_{0}\right)=\mathcal{T}_{k}^{j}\left(E_{11} S\right)\left(E_{k k} \Lambda^{j}+\mathbb{I}_{N}-E_{k k}-E_{11}+E_{11} \Lambda^{-j}\right) W_{0} \\
& =\left(\mathcal{T}_{k}^{j}\left(S_{1 k}\right) E_{1 k} \Lambda^{j}+E_{11} \mathcal{T}_{k}^{j}(S)\left(\mathbb{I}_{N}-E_{k k}-E_{11}\right)+\mathcal{T}_{k}^{j}\left(S_{11}\right) E_{11} \Lambda^{-j}\right) W_{0}, \\
\mathcal{T}_{k}^{j}\left(E_{11} \bar{W}\right) & =\mathcal{T}_{k}^{j}\left(E_{11} \bar{S}\right) \bar{W}_{0}
\end{aligned}
$$

and therefore

$$
\begin{array}{rlrl}
\mathcal{T}_{k}^{j}\left(E_{11} W\right) E_{k^{\prime} k^{\prime}} & \in \mathfrak{g}_{-} W_{0}, & k^{\prime} \neq k, 1, \\
\mathcal{T}_{k}^{j}\left(E_{11} W\right) E_{k k}=\mathcal{T}_{k}^{j}\left(S_{1 k}\right) E_{1 k} \Lambda^{j} W_{0} & \in \mathfrak{g}_{-} W_{0} & & \text { if } j \leq 0, \\
\mathcal{T}_{k}^{j}\left(E_{11} W\right) E_{11}=\mathcal{T}_{k}^{j}\left(S_{11}\right) E_{11} \Lambda^{-j} W_{0} & \in \mathfrak{g}_{-} W_{0} & & \text { if } j>0, \\
\mathcal{T}_{k}^{j}\left(E_{11} \bar{W}\right) & \in \mathfrak{g}_{+} \bar{W}_{0} . & &
\end{array}
$$

Now, we check (67). Notice that

$$
\begin{aligned}
\mathcal{T}_{\bar{k}}^{j}\left(E_{11} W\right) & =\mathcal{T}_{\bar{k}}^{j}\left(E_{11} S\right) \mathcal{T}_{k}^{j}\left(W_{0}\right)=\mathcal{T}_{\bar{k}}^{j}\left(E_{11} S\right)\left(\mathbb{I}_{N}-E_{11}+E_{11} \Lambda^{-j}\right) W_{0} \\
& =\left(E_{11} \mathcal{T}_{\bar{k}}^{j}(S)\left(\mathbb{I}_{N}-E_{11}\right)+\mathcal{T}_{\bar{k}}^{j}\left(S_{11}\right) E_{11} \Lambda^{-j}\right) W_{0}, \\
\mathcal{T}_{\bar{k}}^{j}\left(E_{11} \bar{W}\right) & =\mathcal{T}_{\bar{k}}^{j}\left(E_{11} \bar{S}\right) \mathcal{T}_{\bar{k}}^{j}\left(\bar{W}_{0}\right)=\mathcal{T}_{\bar{k}}^{j}\left(E_{11} \bar{S}\right)\left(E_{k k} \Lambda^{-j}+\mathbb{I}_{N}-E_{k k}\right) \bar{W}_{0} \\
& =\left(E_{11} \mathcal{T}_{\bar{k}}^{j}(\bar{S})\left(\mathbb{I}_{N}-E_{k k}\right)+\mathcal{T}_{\bar{k}}^{j}\left(\bar{S}_{1 k}\right) E_{1 k} \Lambda^{-j}\right) \bar{W}_{0},
\end{aligned}
$$

and therefore

$$
\begin{aligned}
\mathcal{T}_{\bar{k}}^{j}\left(E_{11} W\right) E_{k^{\prime} k^{\prime}} & \in \mathfrak{g}_{-} W_{0}, & k^{\prime} \neq 1, \\
\mathcal{T}_{\bar{k}}^{j}\left(E_{11} W\right) E_{l l}=\mathcal{T}_{\bar{k}}^{j}\left(S_{l l}\right) E_{11} \Lambda^{-j} W_{0} & \in \mathfrak{g}_{-} W_{0}, & \text { if } j>0, \\
\mathcal{T}_{\bar{k}}^{j}\left(E_{1} \bar{W}\right) E_{k^{\prime} k^{\prime}} & \in \mathfrak{g}_{+} \bar{W}_{0}, & k^{\prime} \neq k, \\
\mathcal{T}_{\bar{k}}^{j}\left(E_{11} \bar{W}\right) E_{k k}=\mathcal{T}_{\bar{k}}^{j}\left(\bar{S}_{1 k}\right) \Lambda^{-j} \bar{W}_{0} & \in \mathfrak{g}_{+} \bar{W}_{0}, & \text { if } j \leq 0,
\end{aligned}
$$


Proof of Proposition [6. The proof of these results relies on the previous Lemma 1 and Propositions 2, 5, Let us go into details. We first consider (40). From (66) we find for $k \neq 1$

$$
F\left(\mathscr{M}_{k}, \mathscr{L}_{k}\right)_{>}\left(E_{11} W\right)=F\left(\mathscr{M}_{k}, \mathscr{L}_{k}\right)\left(W_{1 k}\right) E_{1 k}+\mathfrak{g}_{-} W_{0},
$$

so that, as we prove in Proposition [5, we deduce

$$
F\left(\mathscr{M}_{k}, \mathscr{L}_{k}\right)_{>}\left(E_{11} W\right)=E_{11}\left(F(M, L) C_{k k}\right) W+\mathfrak{g}_{-} W_{0}=E_{11}\left(F(M, L) C_{k k}\right)_{+} W+\mathfrak{g}_{-} W_{0} .
$$

Therefore,

$$
R:=F\left(\mathscr{M}_{k}, \mathscr{L}_{k}\right)_{>}\left(E_{11} W\right)-E_{11}\left(F(M, L) C_{k k}\right)_{+} W \in \mathfrak{g}_{-} W_{0}
$$

and from (8) and (66) we get

$$
R g=F\left(\mathscr{M}_{k}, \mathscr{L}_{k}\right)_{>}\left(E_{11} \bar{W}\right)-E_{11}\left(F(M, L) C_{k k}\right)_{+} \bar{W} \in \mathfrak{g}_{+} \bar{W}_{0} .
$$

so that Proposition 2 implies the first formula en (40). Now, from (70) we get for $k \neq 1$

$$
F\left(\overline{\mathscr{M}}_{k}, \overline{\mathscr{L}}_{k}\right)_{>}\left(E_{11} W\right)=F\left(\overline{\mathscr{M}}_{k}, \overline{\mathscr{L}}_{k}\right)\left(W_{1 k}\right) E_{1 k}+\mathfrak{g}_{-} W_{0} .
$$

Hence Proposition 5 ensures that

$$
R:=F\left(\overline{\mathscr{M}}_{k}, \overline{\mathscr{L}}_{k}\right)_{>}\left(E_{11} W\right)-E_{11}\left(F(M, L) C_{k k}\right)_{+} W \in \mathfrak{g}_{-} W_{0}
$$

and from (70) we deduce

$$
R g=F\left(\mathscr{M}_{k}, \mathscr{L}_{k}\right)_{>}\left(E_{11} \bar{W}\right)-E_{11}\left(F(M, L) C_{k k}\right)_{+} \bar{W} \in \mathfrak{g}_{+} \bar{W}_{0} .
$$

In this way, Proposition 2 leads to the last formula in (40). The proof of (41) follows similarly.

\section{Acknowledgements}

The authors wish to thank the Spanish Ministerio de Ciencia e Innovación, research project FIS2008-00200, and acknowledge the support received from the European Science Foundation (ESF) and the activity entitled Methods of Integrable Systems, Geometry, Applied Mathematics (MISGAM). MM wish to thank Prof. van Moerbeke and Prof. Dubrovin for their warm hospitality, acknowledge economical support from MISGAM and SISSA and reckons different conversations with P. van Moerbeke, T. Grava, G. Carlet and M. Caffasso. MM also acknowledges to Prof. Liu for his invitation to visit the China Mining and Technology University at Beijing.

\section{References}

[1] M. Mañas, L. Martínez Alonso, and C. Álvarez-Fernández, The multi-component 2D Toda Hierarchy: discrete flows and string equations arXiv:0809.2720. To be published in Inverse Problems.

[2] K. Ueno and K. Takasaki, Adv. Stud. Pure Math. 4 (1984) 1.

[3] P. Di Francesco, P. Ginsparg and Z. Zinn-Justin, Phys. Rept. 254 (1995) 1.

[4] B. Eynard, An Introduction to Random Matrices, lectures given at Saclay, October 2000, http://www-spht.cea.fr/articles/t01/014/.

[5] L. Bonora and C. S. Xiong, Phys. Lett. B347 (1995) 41.

[6] K. Takasaki, Commun. Math. Phys. 181 (1996) 131.

[7] K. Takasaki and T. Takebe, Rev. Math. Phys. 7 (1995) 743.

[8] P. B. Wiegmann and A. Zabrodin, Comm. Math. Phys. 213 (2000) 523.

M. Mineev-Weinstein, P.B. Wiegmann, and A. Zabrodin, Phys. Rev. Lett. 84 (2000) 5106

I. M. Krichever, M. Mineev-Weinstein, P. B. Wiegmann, and A. Zabrodin, Physica D198 (2004) 1.

I. M. Krichever, A. Marshakov and A. Zabrodin, Comm. Math. Phys. 259 (2005) 1.

M. Mañas, L. Martínez Alonso, and E. Medina, J. Phys. A: Math. Gen. 35 (2002) 401. 
F. Guil, M. Mañas, and L. Martínez Alonso, J. Phys. A: Math. Gen. 36 (2003) 4047.

F. Guil, M. Mañas, and L. Martínez Alonso, J. Phys. A: Math. Gen. 36 (2003) 6457.

L. Martínez Alonso and M. Mañas, J. Math. Phys. 44 (2003) 3294.

M. Mañas, J. Phys. A: Math. Gen. 37 (2004) 9195.

M. Mañas, J. Phys. A: Math. Gen. 37 (2004) 11191.

[9] E. Date, M. Jimbo, M. Kashiwara, and T. Miwa, J. Phys. Soc. Japan 40 (1981) 3806.

M. J. Bergvelt and A. P. E. ten Kroode, Pacific J. Math. 171 23-88 (1995).

M. Mañas, L. Martínez Alonso, and E. Medina, J. Phys.A: Math. Gen. 33 (2000) 2871.

M. Mañas, L. Martínez Alonso, and E. Medina, J. Phys.A: Math. Gen. 33 (2000) 7181.

V. G. Kac and J. W. van de Leur, J. Math. Phys. 44 (2003) 3245.

[10] M. Bertola, B. Eynard and J. Harnad, Comm. Math. Phys. 229 (2002) 73.

[11] M. Bertola, B. Eynard and J. Harnad, Comm. Math. Phys. 243 (2003) 193.

[12] A. B. J. Kuijlaars and K. T-R McLaughlin, J. Comput. Appl. Math. 178 (2005) 313.

[13] M. Adler and P. Van Moerbeke, Comm. Pure and Appl. Math. J. 50 (1997) 241 ; Ann. Math. 149 (1999) 921 .

[14] M. Adler, P. van Moerbeke, and P. Vanhaecke, Commun. Math. Phys. 286 (2009) 1.

M.Adler. J. Delépine and P. van Moerbeke, Comm. Pure and Appl. Math. 62 (2009) 334.

[15] P. M. Bleher and A. B. J. Kuijlaars, Int. Math. Research Notices 2004 (2004) 109; Comm. Math. Phys. 252 (2004) 43; Comm. Math. Phys. 270 (2007) 481.

[16] L. Martínez Alonso, E. Medina, Multiple orthogonal polynomials, string equations and the large-n limit arXiv:0812.3817. To be published in J. Phys. A.

[17] W. Van Assche, J. S. Geronimo, and A. B. J. Kuijlaars, Riemann-Hilbert problems for multiple orthogonal polynomials, In: Special Functions 2000: Current Perspectives and Future Directions (J. Bustoz et al., eds.), Kluwer, Dordrecht, (2001) 23.

[18] E. Daems and A. B. J. Kuijlaars, J. of Approx. Theory 146 (2007) 91.

[19] E. Daems, Asymptotics for non-intersecting Brownian motions using multiple orthogonal polynomials, Ph.D. thesis, K.U.Leuven, 2006, URL http://hdl.handle.net/1979/324.

[20] A.S. Fokas, A.R. Its and A.V. Kitaev, Commun. Math. Phys. 147 (1992) 395.

[21] P. Deift, Orthogonal Polynomials and Random Matrices: A Riemann-Hilbert approach, Courant Lecture Notes in Mathematics Vol. 3, Amer. Math. Soc., Providence R.I. 1999.

[22] P. Deift and X. Zhou, Ann. Math. 2 (1993) 137.

[23] A. Yu Orlov and E. I. Schulman, Lett. Math. Phys. 12 (1986) 171.

[24] I. M. Krichever, Comm. Pure. Appl. Math. 47 (1994) 437.

[25] K. Takasaki, "Dispersionless integrable hierarchies revisited", talk delivered at SISSA at september 2005 (MISGAM program).

[26] K. Takasaki and T. Takebe, Physica D235 (2007) 109.

[27] L. Martínez Alonso, E. Medina and M. Mañas, J. Math. Phys. 47 (2006) 083512.

M. Mañas, E. Medina and L. Martínez Alonso, J. Phys.A: Math. Gen. 39 (2006) 2349. 